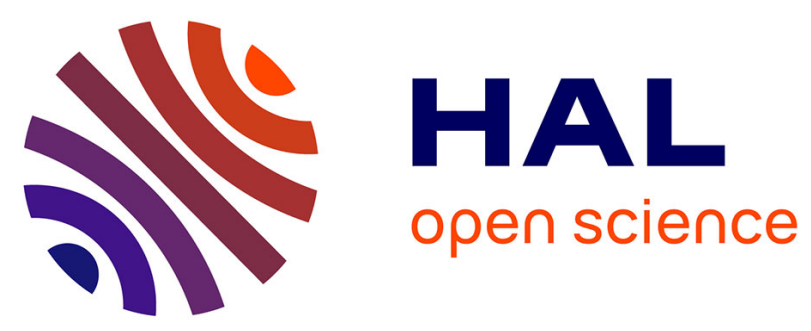

\title{
Properties of lower hybrid solitary structures: A comparison between space observations, a laboratory experiment, and the cold homogeneous plasma dispersion relation
}

P W Schuck, J W Bonnell, Jean-Louis Pinçon

\section{To cite this version:}

P W Schuck, J W Bonnell, Jean-Louis Pinçon. Properties of lower hybrid solitary structures: A comparison between space observations, a laboratory experiment, and the cold homogeneous plasma dispersion relation. Journal of Geophysical Research Space Physics, 2004, 109 (A1), pp.A01310. 10.1029/2002JA009673 . insu-01409739

\section{HAL Id: insu-01409739 \\ https://hal-insu.archives-ouvertes.fr/insu-01409739}

Submitted on 6 Dec 2016

HAL is a multi-disciplinary open access archive for the deposit and dissemination of scientific research documents, whether they are published or not. The documents may come from teaching and research institutions in France or abroad, or from public or private research centers.
L'archive ouverte pluridisciplinaire HAL, est destinée au dépôt et à la diffusion de documents scientifiques de niveau recherche, publiés ou non, émanant des établissements d'enseignement et de recherche français ou étrangers, des laboratoires publics ou privés. 


\title{
Properties of lower hybrid solitary structures: A comparison between space observations, a laboratory experiment, and the cold homogeneous plasma dispersion relation
}

\author{
P. W. Schuck, ${ }^{1}$ J. W. Bonnell, ${ }^{2}$ and J.-L. Pinçon ${ }^{3}$ \\ Received 6 September 2002; revised 20 March 2003; accepted 16 May 2003; published 30 January 2004.
}

[1] Lower hybrid solitary structures have been observed by sounding rockets in the auroral ionosphere for over a decade and a half. Surprisingly, few laboratory experiments have attempted to reproduce this interesting phenomena. Recently, Rosenberg and Gekelman [2001] investigated the interaction between fluctuations near the lower hybrid resonance and field-aligned density striations using the Large Plasma Device at the University of California, Los Angeles. The laboratory measurements of electromagnetic fluctuations localized in a plasma density gradient are new and interesting. This experiment represents another in a series of laboratory investigations that use predictions of the cold homogeneous plasma dispersion to interpret observations of lower hybrid fluctuations interacting with a zero-order plasma density gradient [Bamber et al., 1994, 1995; Rosenberg and Gekelman, 1998]. This experiment is also the first attempt to directly compare laboratory phase velocity estimates with interferometric electric field measurements of lower hybrid solitary structures by the AMICIST, TOPAZ III and PHAZE II sounding rockets [Pinçon et al., 1997; Schuck et al., 1998; Bonnell et al., 1998]. This paper compares the laboratory and space measurements and concludes that they represent completely different phenomena. Furthermore, significant discrepancies between the predictions of the cold homogeneous plasma dispersion relation and the laboratory observations are presented. We speculate that these discrepancies arise because plasma density gradients, essential to description of the laboratory experiment, are neglected in the cold homogeneous plasma dispersion relation. INDEX TERMS: 2487 Ionosphere: Wave propagation (6934); 2471 Ionosphere: Plasma waves and instabilities; 6924 Radio Science: Interferometry; 7831 Space Plasma Physics: Laboratory studies; KEYWORDS: lower hybrid solitary structures, lower hybrid cavities, laboratory experiments, interferometry, density depletions, rotating wave modes

Citation: Schuck, P. W., J. W. Bonnell, and J.-L. Pinçon (2004), Properties of lower hybrid solitary structures: A comparison between space observations, a laboratory experiment, and the cold homogeneous plasma dispersion relation, J. Geophys. Res., 109, A01310, doi:10.1029/2002JA009673.

\section{Introduction}

[2] Laboratory and space experiments are generally complementary. Laboratory experiments excel at studying a single repeatable realization of a phenomena in extraordinary detail. Spacecraft experiments are limited because the payloads move through the plasma while making measurements, can only deploy a limited number of probes, and interact with a localized phenomenon for a limited time-

\footnotetext{
${ }^{1}$ Plasma Physics Division, United States Naval Research Laboratory, Washington, DC, USA.

${ }^{2}$ Space Sciences Laboratory, University of California, Berkeley, Berkeley, California, USA.

${ }^{3}$ Laboratoire de Physique et Chimie de l'Environnement, CNRS, Orleans, France.

Copyright 2004 by the American Geophysical Union. 0148-0227/04/2002JA009673
}

interval. Consequently, space experiments only measure a "projection" of physical quantities onto their trajectory and cannot sample the full spatiotemporal history of a single realization. However, spacecraft experiments compensate for this deficiency by observing many realizations and then building a statistical description of a phenomenon. When consistent statistical pictures emerge, definitive conclusions about phenomena can be drawn despite the absence of a complete space-time history of a single realization. Recently, spacecraft data provided new information about two types of coherent phenomena. Lower hybrid solitary structures (LHSS), the subject of this paper, were discovered and studied in the auroral ionosphere [Labelle et al., 1986; Arnoldy et al., 1992; Kintner et al., 1992; Vago et al., 1992]. Space experiments also determined new information about electron "phase space holes" or "solitary waves." This phenomenon, first described by [Dupree, 1982], received minimal attention by laboratory experiments but is the subject of several statistical studies using spacecraft 
data [Matsumoto et al., 1994; Franz et al., 1998, 2000; Ergun et al., 1998a, 1998b]. Although, laboratory experiments can methodically investigate a particular parameter range in a controlled fashion, these experiments may have difficulty sampling the parameter regime relevant to an unbounded system in space because laboratory devices have limited size, require large magnetic fields to confine highdensity plasmas, and produce ion-electron temperature ratios in a limited regime (usually $T_{\mathrm{i}} / T_{\mathrm{e}} \ll 1$ ).

[3] A few experiments have compared laboratory measurements with sounding rocket and satellite observations of LHSS [Bamber et al., 1994, 1995; Inuzuka et al., 1997; Rosenberg and Gekelman, 1998, 2001; Takeda and Inuzuka, 2000; Kostrov et al., 2001]. However, most laboratory experiments have focused on very general properties of LHSS such as the correlation between enhanced lower hybrid wave activity and density irregularities [Bamber et al., 1994, 1995; Rosenberg and Gekelman, 1998] or the formation of LHSS via wave collapse [Inuzuka et al., 1997; Takeda and Inuzuka, 2000; Kostrov et al., 2001]. We note that space observations have largely discounted wave collapse as a mechanism for the formation of LHSS in the ionosphere [Pécseli et al., 1996, 1997; Kjus et al., 1998; Høymork et al., 2000, 2001; Schuck et al., 2002]. Rosenberg and Gekelman [2001], hereafter denoted RG, represents the first laboratory experiment which performs a detailed comparison between phase velocity estimates in density depletions and sounding rocket interferometric measurements of LHSS. Consequently, RG's experiment is of particular interest.

[4] RG investigate the interaction between fluctuations near lower hybrid resonance and field-aligned density striations using the Large Plasma Device (LAPD) at the University of California, Los Angeles. The data reveal an interesting new mode which exhibits an electromagnetic (transverse) electric field structure perpendicular to the applied magnetic field $\mathbf{B}=B_{0} \hat{z}$ and is localized in the regions of steepest gradient of the density striation. RG compare the laboratory measurements to spacecraft observations of LHSS and conclude that "the laboratory data are consistent with the ionospheric observations" of LHSS by the AMICIST, TOPAZ III, and PHAZE II sounding rockets [Pinçon et al., 1997; Schuck et al., 1998; Bonnell et al., 1998]. Additionally, RG compare the dispersive properties of this mode to the cold homogeneous plasma dispersion relation (CHPDR) and conclude that this cavity mode corresponds to an electrostatic lower hybrid wave despite the electromagnetic nature of the perpendicular electric field structure. Unfortunately, RG's paper contains some conceptual errors regarding the space-based measurements and plasma theory. We disagree with RG's conclusion that "the laboratory data are consistent with the ionospheric observations" of LHSS. The evidence presented by RG indicates that the laboratory measurements and the space observations represent entirely different phenomena. Additionally, we disagree with RG's claim that the laboratory measurements are consistent with the theoretical predictions of the cold homogeneous plasma dispersion relation (CHPDR).

[5] This paper addresses space observations and the LAPD laboratory measurements and is organized as follows. Statistical studies of LHSS will be summarized in section 2. Second, we perform a detailed comparison between the spacecraft and laboratory interferometric measurements and conclude that the space and laboratory measurements correspond to entirely different phenomena. Third, the CHPDR for whistler-lower-hybrid waves is discussed, particularly the nature of the transition from electromagnetic to electrostatic waves; we demonstrate that for the CHPDR this transition occurs for perpendicular wavelengths near the electron collisionless skin depth $k_{\perp} \delta_{\mathrm{e}} \simeq 1$. Fourth, we compare the laboratory mode with the CHPDR and conclude that the laboratory mode is inconsistent with the properties of whistler-lower-hybrid waves described by the CHPDR. Furthermore, the laboratory mode is not in the electrostatic regime of lower hybrid waves either by the wavenumber criteria $k_{\perp} \delta_{\mathrm{e}} \gg 1$ or by the more rigorous ratio of the longitudinal to transverse electric field perpendicular to the applied magnetic field.

\section{Properties of Lower Hybrid Solitary Structures}

[6] RG investigate the interaction of lower hybrid waves with a density striation and compare the results with LHSS observed in the auroral ionosphere. Within this paper the term LHSS describes enhanced wave activity localized in isolated field-aligned cylindrical plasma density striations at frequencies near the lower hybrid resonance of the ambient plasma. Since the first observations by Labelle et al. [1986], this phenomenon has been studied as a possible source of transversely accelerated ions contributing to the overall ion outflow from the auroral ionosphere. Statistical analyses of Freja satellite data demonstrate that the density profiles of LHSS are Gaussian and probably cylindrically symmetric in the plane perpendicular to the geomagnetic field [Pécseli et al., 1996; Kjus et al., 1998; Høymork et al., 2000]. For Freja orbit 1234, the mean 1/e-width of the cavities perpendicular to the magnetic field is about $30 \mathrm{~m}$ with a spread of $\pm 15 \mathrm{~m}$. The mean relative depth of the cavities is $2 \%$ with observations rarely exceeding $10 \%$. LHSS at lower altitude have similar perpendicular scale sizes. However, the TOPAZ III sounding rocket observed significantly deeper striations sometimes approaching $80 \%$. These observations were initially questioned [Ergun et al., 1994, 1995], but the OEDIPUS C sounding rocket has made similar observations of density depletions of 50\% using two independent Langmuir probes: one in ion saturation and the other in electron saturation [Knudsen et al., 1999]. Similarly, PHAZE II sounding rocket observed small-scale plasma density variations of $50 \%$ by tracking changes in the local plasma frequency [McAdams et al., 1998]. The extent of the density striations along the geomagnetic field has not been characterized, but it is certainly several kilometers [Knudsen et al., 1999] and probably tens to hundreds of kilometers.

[7] Pinçon et al. [1997], Schuck et al. [1998], and Bonnell et al. [1998] establish that the wave fronts in the LHSS rotate about the center of the structure using the local frequency-wavenumber-spectrum of the electric field fluctuations. In particular, Bonnell et al. [1998] unambiguously demonstrate that LHSS are composed of right-hand (lefthand) rotating phase fronts at frequencies above (below) the ambient lower hybrid resonance where the handedness is determined with respect to the direction of the geomagnetic field. At a minimum, Pinçon et al. [1997], Schuck et al. 
Table 1. Comparison of Key Plasma Parameters and Ratios for the Laboratory and Ionospheric Experiments ${ }^{\mathrm{a}}$

\begin{tabular}{lccccc}
\hline Key Ratios & AMICIST $^{\mathrm{b}}$ & TOPAZ III $^{\mathrm{c}}$ & PHAZE II $^{\mathrm{d}}$ & Freja $^{\mathrm{e}}$ & LAPD $^{\mathrm{f}}$ \\
\hline$\delta_{\mathrm{e}}, \mathrm{m}$ & 102 & 81 & $80-140^{\mathrm{g}}$ & 230 & 0.0075 \\
$D, \mathrm{~m}$ & 20 & 30 & 30 & 30 & $0.01-0.025$ \\
$f / f_{\mathrm{LH}}$ & $1-4$ & $0.3-4$ & $0.6-2$ & $0.5-3$ & $1.3-6$ \\
$f / f_{\mathrm{pe}}$ & $0.01-0.03$ & $0.0014-0.019$ & $0.003-0.02$ & $0.01-0.05$ & $0.01-0.03$ \\
$f_{\mathrm{p}} f_{\mathrm{ce}}$ & 0.4 & 0.87 & $0.3-0.6^{\mathrm{g}}$ & 0.25 & $1.2-7$ \\
$D / r_{\mathrm{ci}}$ & 5 & 6 & 6 & 20 & $2-15$ \\
$D / \delta_{\mathrm{e}}$ & 0.2 & 0.4 & $-2-0.4^{\mathrm{g}}$ & 0.13 & $1.3-3$ \\
$D / L$ & - & $0.5-2.6^{\mathrm{h}}$ & - & 0.03 & $2-10^{\mathrm{i}}$ \\
$L / r_{\mathrm{ci}}$ & - & - & - & 7.1 & $1-2$ \\
$\lambda_{\|} / \lambda_{\perp}$ & - & - & - & - & $10-100$ \\
$\delta n / n \%$ & - & $10-80$ & & 2 & $10-80$ \\
\hline
\end{tabular}

${ }^{\mathrm{a}}$ Here $f$ is the wave frequency; $f_{\mathrm{LH}}, f_{\mathrm{ce}}, f_{\mathrm{pe}}$ are the lower hybrid resonance, electron cyclotron and electron plasma frequencies respectively; $D$ is the striation diameter (1/e width); $r_{\mathrm{ci}}$ is the majority ion ambient Larmor radius; $L$ is the density gradient scale length; $\lambda_{\perp}$ and $\lambda_{\|}$are the perpendicular and parallel wavelengths; and $\delta_{\mathrm{e}}=c / \omega_{\mathrm{e}}$ is the electron collisionless skin depth.

${ }^{\mathrm{b}}$ Pinçon et al. [1997].

${ }^{\mathrm{c}}$ Schuck et al. [1998].

${ }^{\mathrm{d}}$ Bonnell et al. [1998].

${ }^{\mathrm{e}}$ Average values from Høymork et al. [2000].

${ }^{\mathrm{f}}$ Rosenberg and Gekelman [2001].

${ }^{g}$ Range due to uncertainty in the absolute plasma density.

${ }^{\mathrm{h}}$ Range due to uncertainty in the relative striation depth.

${ }^{\mathrm{i}}$ This range is not consistent with Plate 5 on p. 28,875 of RG.

[1998], and Bonnell et al. [1998] all show that the local phase velocity of spectral components above and below the ambient lower hybrid resonance are antiparallel. Recently, these observations have been confirmed by the Cluster II spacecraft [Tjulin et al., 2003]. Furthermore, Delory [1996] and Delory et al. [1998] show that the perpendicular electric field in the VLF wave bursts (LHSS) observed by the Alaska '93 sounding rocket change from right-hand circularly polarized to linear polarization to left-hand circularly polarized as the experiment traverses the cylindrical density striation.

[8] Using the TOPAZ II search coil magnetometer, Vago et al. [1992] establish that LHSS are essentially an electrostatic phenomenon. Freja observations [Dovner et al., 1994; Eriksson et al., 1994; Pécseli et al., 1996; Kjus et al., 1998; Høymork et al., 2000] consistently support this conclusion. On p. 18,528, Høymork et al. [2000] state that "Only very small magnetic fields are observed, and they show little or no correlation with the variations in electric field;" Dovner et al. [1994] report $E / B \gtrsim 8 \times 10^{7} \mathrm{~m} / \mathrm{s}$, and Kjus et al. [1998] report $E / B \gtrsim 10^{6} \mathrm{~m} / \mathrm{s}$. All three works conclude that these fluctuations are electrostatic. Since $E$ and $B$ are uncorrelated in these measurements, the value of $E / B \sim$ $10^{6}-10^{8} \mathrm{~m} / \mathrm{s}$ represents a lower bound for this ratio. Generally, the raw $E / B$ ratio cannot be used exclusively to conclude that the fluctuations on the whistler-lower-hybrid branch are electrostatic particularly when this ratio is less than the speed of light $c \sim 3 \times 10^{8} \mathrm{~m} / \mathrm{s}$. However, Eriksson et al. [1994] presents spectra for LHSS where $E / B>7.5 c$ at frequencies above the ambient lower hybrid resonance. This last observation provides the most convincing quantitative evidence using the $E / B$ ratio that LHSS are electrostatic. Although as section 4 demonstrates, the perpendicular scale length of the phenomena is perhaps a more rigorous criterion for concluding that fluctuations on the whistlerlower hybrid dispersion branch are electrostatic.

[9] Table 1 is a comparison of key plasma parameters and ratios for the laboratory and ionospheric experiments. The values of key plasma parameter ratios for the Large Plasma Device (LAPD) experiment generally compare favorably to the ionospheric parameters. However, the ratio of the striation diameter to the electron collisionless skin depth $D / \delta_{\mathrm{e}}$ is $1-3$ in the laboratory and $0.1-0.4$ in the ionospheric observations. The disparity between the laboratory results and the ionospheric observations described later in this paper may depend critically upon this parameter.

\section{Comparison Between Sounding Rocket Observations and the Laboratory Experiment}

[10] Pinçon et al. [1997], Schuck et al. [1998], and Bonnell et al. [1998] estimate the local dispersion relation of electric field fluctuations within LHSS using local frequency-wavenumber-spectra formulated on the Morlet wavelet basis. The wavelet basis relaxes the restriction of stationarity usually assumed in traditional Fourier analysis. This formulation is necessary because LHSS waves are nonstationary in the spacecraft frame although the density cavities are localized and nearly stationary in the rest frame of the plasma [Dovner et al., 1994; Lynch et al., 1996; Knudsen et al., 1998; Høymork et al., 2000]. The local frequency-wavenumber-spectrum provides an estimate of the wavenumber $k_{d}=\mathbf{k} \cdot \hat{\mathrm{d}}$ and the phase velocity $V_{p} \simeq$ $\omega / k_{d}$ along the direction of the interferometer axis $d \equiv \mathbf{d} / \mathbf{d}$ by constructing a power weighted histogram as a function of frequency and phase of the wavelet cross-spectrum measured between two collinear electric field antennas separated by a distance $d$.

[11] Figure 1 shows a schematic diagram of the electric field measurements made by sounding rocket interferometers and the LAPD experiment along trajectories across a cylindrical density striation. The trajectories for each situation are denoted by dotted lines and the direction of the phase velocity determination is denoted by the arrows labeled $V_{\mathrm{p} \|}$ or $V_{\mathrm{p} \perp}$. The extent of the density striation is indicated by the thick circular line. Direct comparison of the sounding rocket and laboratory electric field interferometric measurements is complicated by the differences in data gathering techniques between the two experiments, and fundamental differences 


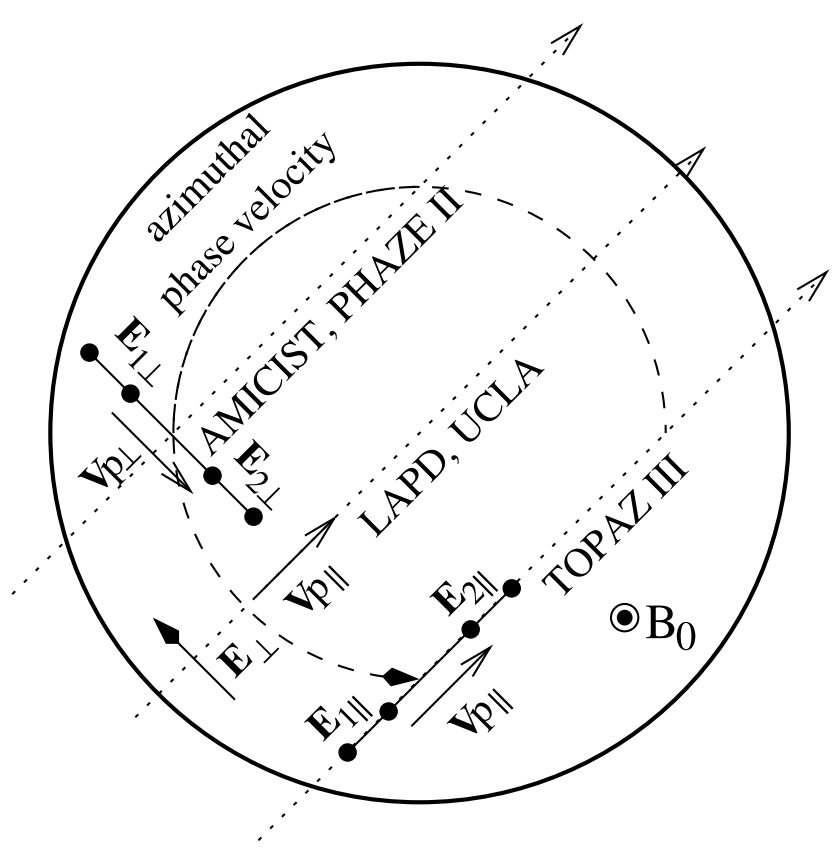

Figure 1. A schematic diagram of the electric field measurement orientations for sounding rocket interferometers and the LAPD experiment along trajectories across a cylindrical density striation. The trajectories for each situation are denoted by dotted lines and the direction of the phase velocity determination is denoted by the arrows labeled $V_{\mathrm{p} \|}$ or $V_{\mathrm{p} \perp}$. The extent of the density striation is indicated by the thick circular line. A "right-handed" azimuthal phase velocity is indicated by the dashed arc and the geomagnetic field points out of the page.

in electric field structure for the two situations. Particularly, the laboratory experiment always determines the phase velocity of the azimuthal electric field in the direction parallel to the trajectory across the density striation whereas the sounding rocket experiments perform different phase velocity estimates which depend on the orientation of the electric field antenna, the interferometer axis, and the payload velocity vector. The important differences between the ionospheric and laboratory experiments and observations will be discussed in detail below.

[12] Figure 1 shows that to lowest order, the AMICIST [Pinçon et al., 1997] and PHAZE II [Bonnell et al., 1998] sounding rocket experiments determine the phase velocity perpendicular to the trajectory, of electric field fluctuations perpendicular to the trajectory. These observations show that the phase velocity of the electric field determined along the interferometer axis changes direction as spacecraft in this orientation cross LHSS. The relative orientation between the interferometer and the geomagnetic field combined with the statistical conclusion that LHSS are probably cylindrical [Pécseli et al., 1996; Høymork et al., 2000, 2001], provides information about the direction the azimuthal phase velocity within the cylindrical density structure. The phase fronts exhibit right-hand or left-hand rotation (with respect to the geomagnetic field) about the center of the LHSS. The AMICIST data unambiguously demonstrate that the reversal in phase velocity is consistent with right-hand rotating waves above the ambient lower hybrid resonance [Pinçon et al., 1997]. Unfortunately, AMICIST did not detect geophysical waves at frequencies below the ambient lower hybrid resonance. Figure 1 shows a "right-handed," with respect to the geomagnetic field $\mathbf{B}=$ $B_{0} \hat{z}$, azimuthal phase velocity indicated by the dashed arc. This right-hand rotating wave mode exhibits a positive phase $V_{\mathrm{p} \perp}>0$ velocity along the interferometer axis during the first half of the event and a negative phase velocity $V_{\mathrm{p} \perp}<0$ during the second half of the event, i.e., the phase velocity along the interferometer axis changes direction as the payload crosses the LHSS. Using a similar analysis with the PHAZE II data, Bonnell et al. [1998] unambiguously demonstrate the simultaneous existence of right-hand rotating waves above the ambient lower hybrid resonance and left-hand rotating waves below the ambient lower hybrid resonance.

[13] The AMICIST and PHAZE II observations would not be convincing if these results corresponded to exceptional cases. However, these results are independent of the trajectory or chord across the LHSS event. For example, if the AMICIST/PHAZE II (upper) trajectory shown in Figure 1 occurred along the TOPAZ III (lower) trajectory, the phase velocity of a right-hand rotating mode would change in precisely the same manner. The same is true for a trajectory along the diameter of the striation. This independence is substantiated by observations. Pinçon et al. [1997] examine nine distinct LHSS with eight corresponding to rotating waves. The ninth does not contradict these results but is inconclusive due to the "complexity of its internal structure (several characteristic scale lengths for a given frequency band)" [Pinçon et al., 1997, p. 17,291]. Bonnell et al. [1998] examine 22 events which exhibit counterrotating modes above and below the ambient lower hybrid resonance and other events which exhibit only right-hand rotating modes above the ambient lower hybrid resonance. There is sufficient statistical evidence to support the observational conclusions by Pinçon et al. [1997] and Bonnell et al. [1998], Delory et al. [1998], and Tjulin et al. [2003] have confirmed these deductions.

[14] Figure 1 shows that to lowest order, the TOPAZ III sounding rocket experiment [Pinçon et al., 1997; Schuck et al., 1998] determines the phase velocity parallel to the trajectory, of electric field fluctuations parallel to the trajectory. These observations show that for a fixed frequency band, the direction of the phase velocity along the interferometer axis remains constant as spacecraft with this orientation cross LHSS. Furthermore, the waves at frequencies above the ambient lower hybrid resonance propagate along the interferometer antiparallel to the waves at frequencies below the ambient lower hybrid resonance. This is consistent with the AMICIST and PHAZE II observations. Figure 1 shows that a right-hand rotating wave mode exhibits a positive phase velocity along the interferometer axis during the entire TOPAZ III trajectory across the LHSS. Similarly, a left-hand rotating wave mode exhibits a negative phase velocity for the same trajectory. If the TOPAZ III (lower) trajectory shown in Figure 1 occurred along the AMICIST/ PHAZE II (upper) trajectory, the results are equivalent except the signs of measured phase velocities above and below the ambient lower hybrid resonance are interchanged. This degeneracy in the absolute sign of the measured phase velocity prevents an unambiguous determination of the 
absolute direction of rotation for this interferometer orientation. However, one may still correctly conclude from observational data that a mode is rotating.

[15] Since the sounding rockets with the same interferometer orientation consistently observe the same behavior in the phase velocity, the results are statistically independent of how experiments intersect a density striation. Although the linear theory of LHSS proposed by Seyler [1994], Schuck et al. [1998], and Schuck [1999] provides an explanation for the observed behavior, this theory is not necessary to conclude that the phase fronts of the electric field fluctuations are rotating within the LHSS; this conclusion was reached solely on the observational data.

[16] When the laboratory electric field measurements are considered within the context of the sounding rocket interferometric measurements, the difference between the phase velocity estimates is apparent. The laboratory experiment determines the phase velocity parallel to a trajectory of the azimuthal electric field $E_{\theta}$. This estimate is derived from the careful spatial analysis of the direction and phase of the electric field within the laboratory striation. $E_{x}$ and $E_{y}$ are measured at each point in the $x y$-plane and the radial $E_{r}$ and azimuthal $E_{\theta}$ components are computed with respect to center of the cylindrical geometry. The electric "fields in the laboratory are almost entirely tangential" to the cylindrical density striation and the magnitude is largest within the gradient of the plasma density. Therefore the "field is primarily azimuthal" and the radial electric field is small $E_{r} \approx 0[\mathrm{RG}$, p. 28,880$]$. At each point in the $x y$-plane the phase $\psi$ of the azimuthal electric field is computed. Plate 9 on p. 28,882 and Figure 7 on p. 28,883 of RG show that $\psi$ "does not vary much azimuthally," except near the paddle support (see below). However, $\psi$ " does vary significantly along the radial cut" [RG, p. 28,880]. The local wavenumber and phase velocity can be estimated from $\mathbf{k}=\nabla \psi$ and $\mathbf{V}_{\mathrm{p}}=\omega / \mathbf{k}$, respectively.

[17] For the laboratory results reported, the phase velocity is always determined from the gradient in the phase parallel to the trajectory. RG consider two trajectories through the striation: one along an azimuthal path and another along a diameter. The azimuthal path establishes that the phase of $E_{\theta}$ does not change significantly around the density striation at this radius because this trajectory avoids the distinct azimuthal asymmetry introduced by the paddle support at $(x, y)=$ $(0.25,0.5-1) \mathrm{cm}$ (see Plate 9 in $\mathrm{RG})$. This path is not relevant for the interpretation of sounding rocket data because the sounding rockets do not orbit LHSS; even segments of this path have little relevance to the sounding rocket measurements because the gradient of phase is zero in the azimuthal direction and thus the phase velocity along this trajectory is ill-defined in the laboratory. The path along the diameter is somewhat pertinent to the interpretation of sounding rocket data. For this radial path the laboratory experiment determines the phase velocity, parallel to the trajectory $\mathbf{V}_{\mathrm{p} \|}$, of electric field fluctuations $\mathbf{E}_{\perp}=E_{\theta} \hat{\theta}$ perpendicular to the trajectory. Figure 7 of RG shows that the radial gradient in the phase $\psi$ of $E_{\theta}$ changes sign along the diameter of the striation leading to the conclusion that the phase velocity changes sign along this trajectory. Since the AMICIST and PHAZE II sounding rockets observe a qualitatively similar behavior for the phase velocity within LHSS, RG conclude that "the laboratory

Table 2. Orientation of the Electric Field and Phase Velocity Measured in the Laboratory and Ionospheric Experiments ${ }^{\mathrm{a}}$

\begin{tabular}{|c|c|c|c|}
\hline Experiment & $\begin{array}{l}E \text {-Field } \\
\text { Measured } \\
\| / \perp \text { to } \\
\text { Trajectory }\end{array}$ & $\begin{array}{c}\text { Phase Velocit } \\
\text { Determined } \\
\| / \perp \text { to } \\
\text { Trajectory }\end{array}$ & $\begin{array}{c}\text { Properties } \\
\text { of the Phase } \\
\text { Velocity }\end{array}$ \\
\hline LAPD & $\perp$ & $\|$ & changes sign along diameter \\
\hline AMICIST/PHAZE I & $\perp$ & $\perp$ & changes sign \\
\hline TOPAZ III & $\|$ & $\|$ & constant \\
\hline
\end{tabular}

${ }^{\mathrm{a}}$ The electric field and phase velocity are characterized according to their direction relative to trajectory/path across the cylindrical density striation. See also Figure 1.

data are consistent with the ionospheric observations" $[\mathrm{RG}$, p. 28,882]. However, Figure 1 shows that the two sounding rocket interferometer orientations used to determined the phase velocity of LHSS waves are quite different from this laboratory estimate. The orientations and observations of the laboratory and sounding rocket experiments are summarized in Table 2. Any qualitative correspondence between the space and laboratory measurements is irrelevant, since no combination of electric field component and phase velocity direction from the sounding rocket experiment is represented in the published laboratory data.

[18] RG also comment on other trajectories across the laboratory density striation:

\begin{abstract}
By traversing the striation over several different chords we have found that the direction of the measured phase velocity is heavily dependent on the path taken. Along some paths these phase shifts appear as phase velocities which are constant across the striation; along other paths the phase velocity appears to change sign across the striation. Pinçon et al. [1997] and Bonnell et al. [1998] show that the electric field phase velocity reverses across the striation. In the work of Schuck et al. [1998] the phase velocity remains constant. In other words, the laboratory data are consistent with the ionospheric observations, but to completely understand the phase shifts, it may be necessary to plot the threedimensional phase fronts. [RG, pp. 28,880-28,882]
\end{abstract}

[19] These measurements along unspecified chords are more difficult to interpret and directly compare with the ionospheric observations because the laboratory density striation exhibits a significant azimuthal asymmetry at the top of the density striation near $(x, y)=(0.25,0.5-1.5) \mathrm{cm}$ (see Plate 1 in RG). RG state on p. 28,879 that this asymmetry "is caused by the support for the paddle used to create the striation." The laboratory data also exhibits an asymmetry in the amplitude and direction of the electric field in Plates 2, 4, and 8 and, in particular, the phase of the electric field in Plate 9 near $(x, y)=(0.25,0.5-1) \mathrm{cm}$. The paddle support also produces these asymmetries because

\footnotetext{
The density dependence of the waves warps the axial phase fronts. A phase shift measurement in a plane perpendicular to the background magnetic field then will measure shifts proportional to the effect of the plasma inhomogeneity. [RG, p. 28,880]
}

[20] Consequently, an asymmetry in the density profile will produce a corresponding asymmetry in the electric field quantities. These asymmetries cause the path dependence in the direction of the phase velocity for the laboratory experiment. Indeed, when the phase velocity is determined parallel to a straight trajectory, a cylindrically symmetric 
phase profile $\psi=\psi(r)$ always exhibits at least one reversal in the direction of this phase velocity. Thus for a cylindrically symmetric phase profile, there are no paths that do not exhibit a reversal in the direction of this phase velocity, nor any path dependence as described in the laboratory observations. Obviously, the phase velocity determined from data along a chord passing directly through the asymmetries generated by the support for the paddle will exhibit different properties than measurements along a chord avoiding them. This asymmetry is the main cause for the path dependence in the direction of the phase velocity determined from laboratory data along different chords; some chords exhibit a reversal in the direction of the phase velocity and others do not. This path dependence is not manifest in the ionospheric data. If the asymmetries in field and phase distributions observed in the laboratory directly reflected the electric field structure of LHSS, then the path dependence for the direction of phase velocity described by RG would also be observed in space data. Instead, the direction of the phase velocity in LHSS exhibits well-ordered patterns determined solely by the orientation of the interferometer rather than the particular path. In addition, Freja multiprobe measurements of LHSS convincingly demonstrate that the associated density profile is surprisingly Gaussian and symmetric along the satellite trajectory [Høymork et al., 2000, 2001]. These data are consistent with a cylindrically or (worst case) elliptically symmetric Gaussian density profile in the plane perpendicular to the geomagnetic field. Thus the path dependence caused by asymmetries in the laboratory density profile is not relevant to, nor exhibited by, the ionospheric observations of LHSS.

[21] Regardless of the difficulties in directly comparing the laboratory and space observations and despite the lack of direct correspondence between the laboratory and sounding rocket phase velocity estimates, one might still hypothesize that the general electric field structure of the mode observed in the laboratory experiment is equivalent to LHSS in space. A direct comparison between the LHSS observations and the laboratory measurements is biased against the laboratory data because the laboratory analysis exhibits asymmetries which lead to a path dependent phase velocity that is not observed in the LHSS in space. However, this hypothesis can be tested by considering what each of the interferometer orientations (AMICIST and PHASE-II or TOPAZ-III) would observe while crossing an electric field model for an idealized laboratory striation without the azimuthal asymmetries discounted above. Here, the general behavior of the laboratory mode is the focus of investigation rather than the particular details of the irrelevant asymmetries introduced by the paddle support. Since these laboratory asymmetries are deleterious to a fair comparison between the laboratory and ionospheric observations, the laboratory data must be modeled using the dominant, azimuthally symmetric modal structure. As suggested by RG on p. 28,882 and exhibited by Figure 7 and Plate 9 in RG, the phase of the dominant azimuthal electric field $E_{\theta}$ in the laboratory striation can be described (to lowest order) by the azimuthally symmetric distribution $\psi=$ $\psi(r)$ peaked at the center of the striation shown in the upper panel of Figure 2.

[22] The radial phase velocity of $E_{\theta}$ changes sign along the diameter of the laboratory striation. However, a phase
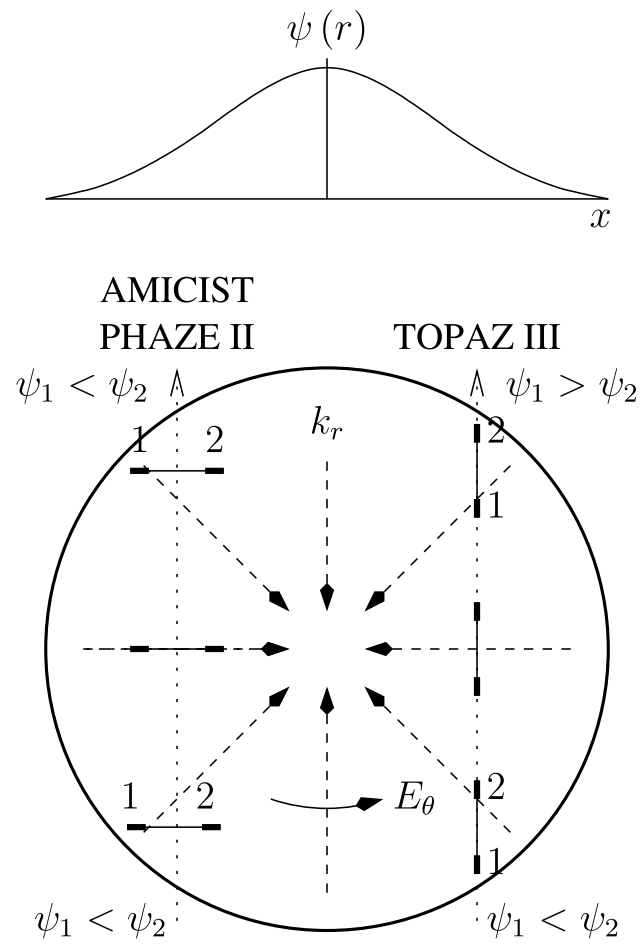

Figure 2. (top) The idealized spatial dependence of the phase $\psi$ of the azimuthal electric field $E_{\theta}$ in the laboratory density striation. (bottom) A schematic representation for the trajectories of the two different electric field interferometers through the laboratory density striation. The direction of the gradient in the phase $k_{r}=\partial_{r} \psi(r)$ of the azimuthal electric field $E_{\theta}$ is shown as dashed arrows, the trajectories of the interferometers are shown as dotted lines along chords of the striation, and the collinear electric field antennas of the interferometers, labeled 1 and 2, are shown as thick lines.

velocity measurement by either of the two space interferometer orientations is poorly defined along this trajectory. For the AMICIST orientation, the phase shift is insignificant because $k_{\theta} \approx 0$. For the TOPAZ III orientation the electric field measurement is insignificant because $E_{\mathrm{r}} \approx 0$. Furthermore, such a radial crossing is unlikely in space. Consequently, we consider what the spacecraft interferometers would detect along a chord across the laboratory striation.

[23] The lower panel of Figure 2 shows a schematic representation of the two different spacecraft interferometer orientations on trajectories through the electric field structure of the laboratory striation. The direction of the gradient in the phase $\psi$ of the azimuthal electric field $E_{\theta}$ is indicated by dashed arrows, the trajectories of the interferometers are shown as dotted lines along chords of the striation, and the electric field antennas of the interferometers are shown as thick lines and are labeled 1 and 2. The trajectory on the left-hand side of the figure corresponds to the AMICIST and PHAZE II measurements where the interferometer axis is perpendicular to the payload velocity whereas the trajectory on the righthand side corresponds to the TOPAZ II measurements, 
where the interferometer axis is parallel to the payload velocity.

[24] For the AMICIST and PHAZE II flight configuration (left side), antenna 2 is always closer to the center of the striation than antenna 1 . Thus $\psi_{2}>\psi_{1}$ always and the direction of phase velocity does not change while crossing the striation for this antenna orientation. This is opposite to the behavior exhibited by the LHSS data from the AMICIST and PHAZE II sounding rockets. For the TOPAZ III flight configuration (right side), antenna 2 is initially closer to the center of the striation than antenna 1 , and so the phase of the electric field measured by antenna 2 is larger than that measured by antenna $1\left(\psi_{2}>\psi_{1}\right)$. After crossing the striation, antenna 1 is closer to the center of the striation than antenna 2, and so the phase of the electric field measured by antenna 1 is greater than that measured by antenna $2\left(\psi_{2}<\psi_{1}\right)$. Thus the projection of the phase velocity along the interferometer axis changes direction while crossing the striation for this antenna orientation. Again, this is opposite to the behavior exhibited by the LHSS data from the TOPAZ III sounding rocket. Consequently, the hypothesis that the electric field structure is the same in LHSS and in laboratory experiment is false. The evidence indicates that the space and laboratory measurements represent entirely different phenomena.

[25] Another point of comparison between the laboratory experiment and the ionospheric observations is the distribution of the electric field power along trajectories across the density striations in the laboratory and ionosphere. Satellites and sounding rockets often observe single and/ or double-humped distributions of electric field power in the temporal domain as the spacecraft cross LHSS. Indeed, RG point out that a

\begin{abstract}
recent analysis of Freja data Kjus et al. [1998] discusses the basic statistical characteristics of lower hybrid waves and associated density depletions. In the observational part of their paper they state that in "approximately $10 \%$ of all the cases" the wave energy has a double humped appearance as the satellite flew through the striation. On the basis of the data they accumulated Kjus et al. [1998, p. 26,644] speculate that "most of the cavities are actually associated by a ring distribution of wave energy... and the observations without any bifurcation are merely a consequence of the satellite glancing the cavity along a trajectory, avoiding the central wave energy depletion." In our experiments the wave energy is localized on the density gradient and a fly [sic] through the cavity would lead to a double hump in wave energy as well [RG, p. 28,883].
\end{abstract}

[26] However, the ionospheric observations of single and double-humped power distributions provide additional details about this phenomenon. Plate 1 in the work of Pinçon et al. [1997] shows the simultaneous observation of single and double-humped distributions of wave energy at $12 \mathrm{kHz}$ and $6 \mathrm{kHz}$ on a trajectory through a single LHSS event from the AMICIST experiment. The frequency-wavenumber spectral analysis of the data shown in Plate 2 of Pinçon et al. [1997] reveals that these two power distributions are associated with different scale lengths: $45 \mathrm{~m}$ and $16.7 \mathrm{~m}$, respectively. The scale length $l=2 \pi d / \Delta \psi$ is determined from the phase shift $\Delta \psi$, measured along the length $d$ of interferometer axis.

[27] The characteristics of the wave modes in the AMICIST LHSS event are summarized in Table 3. These results indicate that there are two different wave modes
Table 3. Wave Mode Characteristics of a Single LHSS Event From the AMICIST Experiment [Pinçon et al., 1997]

\begin{tabular}{ccc}
\hline Frequency, $\mathrm{kHz}$ & Scale, $\mathrm{m}$ & Temporal Distribution \\
\hline 12 & 45 & single-humped \\
6 & 16.7 & double-humped \\
\hline
\end{tabular}

comprising this LHSS and the scale length and frequency of each mode is different. The scale lengths of the modes detected in the space experiment are dependent on frequency and not exclusively determined by the cavity diameter. In contrast, the perpendicular wavenumber of the laboratory mode is set by the diameter of the density striation, and this scaling is independent of frequency [RG, p. 28,882]. Consequently, RG's observation of a double-humped distribution of power along a trajectory across the density striation is not sufficient grounds for associating the laboratory measurements with LHSS. We note that an interferometric analysis of a numerical simulation based on an electrostatic nonlinear fluid model reproduced this LHSS event with remarkable accuracy; including the frequencies, wavenumbers, and the asymmetry in the frequency-wavenumberspectrum due to the payload orientation (see Plates 2, 3, and 4 in the work of Pinçon et al. [1997]).

\section{Cold Homogeneous Plasma Dispersion Relation (CHPDR) and Whistler-Lower-Hybrid Waves}

[28] Frequently, the cold homogeneous plasma dispersion relation (CHPDR) is useful for the interpretation of space and laboratory data. The CHPDR provides a theoretical framework for mode identification based on the predicted relationship between frequency $\omega$ and wavevector $\mathbf{k}$ and the observed field polarizations and ratios as functions of $\omega$ and k. Nevertheless, when significant inhomogeneities exist in the plasma this interpretation must proceed cautiously, because inhomogeneities can change the nature and propagation characteristics of the homogeneous plasma modes and introduce new modes of propagation which have no correspondence to the homogeneous plasma theory.

[29] RG investigate the characteristics of waves near the lower hybrid frequency propagating in a sharp plasma density depletion. They compare the laboratory data with dispersion curves computed from the theoretical CHPDR. We note that equations (1)-(3) appearing in RG do not correspond to the CHPDR but rather conform to the "Altar-Appleton-Hartree" dispersion relation (AAHDR) (termed the "Appleton-Hartree" dispersion relation in RG). [Appleton, 1928, 1932; Hartree, 1931; Gillmor, 1982]. (Gillmor [1982] discusses E. V. Appleton's collaboration with Wilhelm Altar. The appendix of Gillmor [1982] presents Altar's 1926 draft manuscript which derives the AHHDR.) The AAHDR neglects ion dynamics and describes cold-plasma electromagnetic electron modes. This approximate dispersion relation does not describe electrostatic lower hybrid waves because the AAHDR neglects the ion inertial response perpendicular to the applied magnetic field $\mathbf{B}_{0}$ which is essential to the lower hybrid mode. (The literature occasionally suggests that the AAHDR retains ion dynamics. We speculate that 
this confusion is either due to the historical terminology "magneto-ionic theory" associated with the AAHDR or the ambiguous statements by Krall and Trivelpiece [1973] and others.) Despite RG's error in presentation, the correct CHPDR is used in their numerical computation of the dispersion curves. RG conclude the cavity mode in the laboratory experiment is a lower hybrid wave based on the "very good agreement between theoretical and measured values" of wavenumber [RG, p. 28,880]. Consequently, some discussion of the CHPDR is necessary because this theoretical dispersion relation is an essential element of RG's mode identification analysis.

[30] The CHPDR provides an accurate two-fluid description of lower hybrid waves propagating in a zero temperature, uniform plasma comprised of ions and electrons. The predictions of the CHPDR are often remarkably accurate even when the plasma is not cold or uniform. Following Stix [1992, pp. 7-9], the vector wave equation for the CHPDR is

$$
\left(\begin{array}{ccc}
S-\eta^{2} \cos ^{2} \theta & -i D & \eta^{2} \cos \theta \sin \theta \\
i D & S-\eta^{2} & 0 \\
\eta^{2} \sin \theta \cos \theta & 0 & P-\eta^{2} \sin ^{2} \theta
\end{array}\right)\left(\begin{array}{l}
E_{x} \\
E_{y} \\
E_{z}
\end{array}\right)=0
$$

where

$$
\begin{gathered}
S=\frac{1}{2}(R+L), \\
D=\frac{1}{2}(R-L), \\
R=1-\frac{\omega_{\mathrm{e}}^{2}}{\omega\left(\omega-\Omega_{\mathrm{e}}\right)}-\frac{\omega_{\mathrm{i}}^{2}}{\omega\left(\omega+\Omega_{\mathrm{i}}\right)}, \\
L=1-\frac{\omega_{\mathrm{e}}^{2}}{\omega\left(\omega+\Omega_{\mathrm{e}}\right)}-\frac{\omega_{\mathrm{i}}^{2}}{\omega\left(\omega-\Omega_{\mathrm{i}}\right)}, \\
P=1-\frac{\omega_{\mathrm{e}}^{2}+\omega_{\mathrm{i}}^{2}}{\omega^{2}} .
\end{gathered}
$$

A nontrivial solution exists if and only if the determinant of the $3 \times 3$ matrix is zero which leads to the dispersion relation

$$
k_{\perp}^{2} / k_{z}^{2}=\tan ^{2} \theta=-\frac{P\left(\eta^{2}-R\right)\left(\eta^{2}-L\right)}{\left(S \eta^{2}-R L\right)\left(\eta^{2}-P\right)} .
$$

[31] Above, $\eta=k c / \omega$ is the refractive index, $\theta$ is the angle between the wave-vector $\mathbf{k}$ and the magnetic field $\mathbf{B}_{0}=B_{0} \hat{z}$, $\omega_{j}^{2}=e^{2} n /\left(\epsilon_{0} m_{j}\right), \Omega_{j}=e B_{0} / m_{j}$, and $m_{j}$ with $(j=\mathrm{i}, \mathrm{e})$ are the species plasma frequency, cyclotron frequency, and mass, respectively, $n$ is the plasma density, $\omega$ is the wave frequency, $c$ is the speed of light, $e$ is the elemental unit of charge, and $\epsilon_{0}$ is the permittivity of free space. The AAHDR can be obtained from equations (1)-(2) by neglecting the ion a

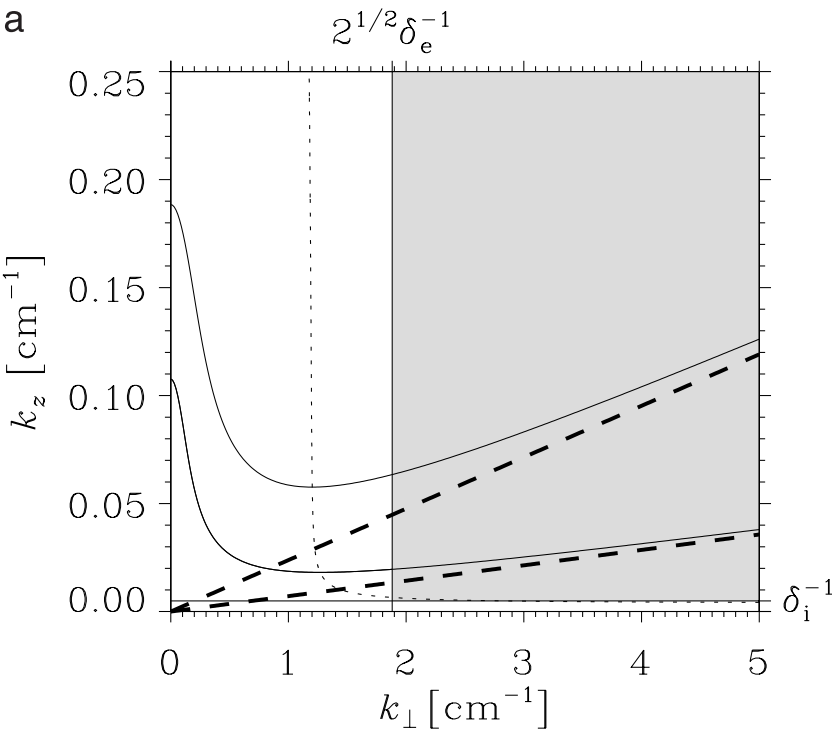

b

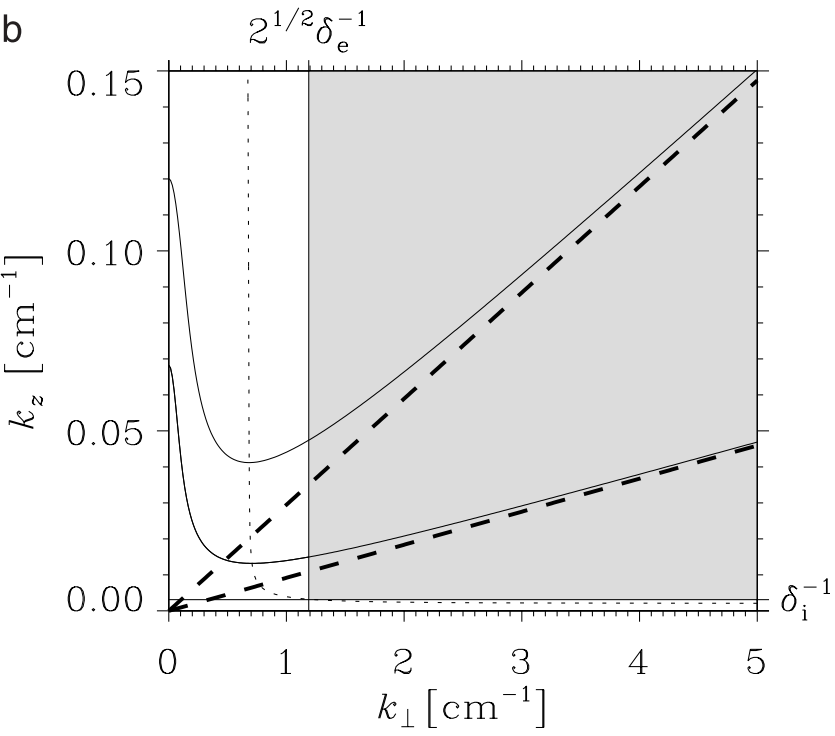

Figure 3. Relationship between $k_{z}$ and $k_{\perp}$ determined from the CHPDR for conditions corresponding to the laboratory experiment for $n_{0}=5 \times 10^{11} \mathrm{~cm}^{-3}$ (a) and $n_{0}=2 \times 10^{11} \mathrm{~cm}^{-3}$ (b) at the frequencies $f=90 \mathrm{MHz}$ (upper solid lines) and $f=$ $30 \mathrm{MHz}$ (lower solid lines). The dashed lines denote the electrostatic dispersion relation (equation (4b)) or resonance cone. The approximate range of validity for the electrostatic limit is indicated by the shaded region determined from equation (4a). $\left(k_{\perp}>\sqrt{2} \delta_{\mathrm{e}}^{-1}, k_{z}>\delta_{\mathrm{i}}^{-1}\right)$. The light dashed line corresponds to the wavenumbers of the Gendrin mode where the perpendicular group velocity is zero on the whistlerlower-hybrid dispersion curve.

motion $\left(\Omega_{\mathrm{i}} \rightarrow 0, \omega_{\mathrm{i}} \rightarrow 0\right)$ in the Stix parameters $\mathrm{R}, \mathrm{L}$, and $P$ described by equations (1a)-(1f).

\section{1. "Electrostatic" and "Electromagnetic" Approximations}

[32] Figure 3 shows the relationship between $k_{z}$ and $k_{\perp}$ determined from the exact CHPDR for whistler-lowerhybrid waves in an Argon plasma with a background magnetic field of $1650 \mathrm{G}$ corresponding to the laboratory 
experiment. Figure 3 a corresponds to the plasma density external to the striations $n_{0}=5 \times 10^{11} \mathrm{~cm}^{-3}$ and Figure $3 \mathrm{~b}$ corresponds to the plasma density at the minimum of the striations $n_{0}=2 \times 10^{11} \mathrm{~cm}^{-3}$ (striation 1 is somewhat deeper $n_{0} \sim 1 \times 10^{11} \mathrm{~cm}^{-3}$ ). The upper and lower solid lines correspond to the two frequencies, $f=90 \mathrm{MHz}$ and $f=$ $30 \mathrm{MHz}$, respectively. The group velocity is normal to the curves of the CHPDR for constant frequency and points towards higher frequencies [Poeverlein, 1948]. The light dashed line corresponds to the wavenumbers of the Gendrin mode where the perpendicular group velocity is zero on the whistler-lower-hybrid dispersion curve [Gendrin, 1961; Sturrock, 1962].

[33] Approximating equation (1) with $\omega \ll \omega_{\mathrm{e}}, \Omega_{\mathrm{i}} \ll \omega$ and expanding $R$ and $L$ to order $\omega / \Omega_{\mathrm{e}}$ to account for the electron polarization drift, an accurate description of the cold homogeneous whistler-lower-hybrid dispersion relation is

$$
\omega^{2} \approx \frac{\Omega_{\mathrm{e}}^{2} \omega_{\mathrm{i}}^{4}\left(1+k^{2} \delta_{\mathrm{i}}^{2}\right)\left(1+k^{2} \delta_{\mathrm{e}}^{2}+k_{z}^{2} \delta_{\mathrm{i}}^{2}\right)}{\omega_{\mathrm{e}}^{4}-k_{\perp}^{2} \delta_{\mathrm{e}}^{2} \omega_{\mathrm{e}}^{2} \Omega_{\mathrm{e}}^{2}+\left(1+k^{2} \delta_{\mathrm{i}}^{2}\right)\left(2+k_{\perp}^{2} \delta_{\mathrm{e}}^{2}\right) \omega_{\mathrm{i}}^{2} \Omega_{\mathrm{UH}}^{2}}
$$

where $\Omega_{\mathrm{UH}}^{2}=\omega_{\mathrm{e}}^{2}+\Omega_{\mathrm{e}}^{2}$ is the upper hybrid resonance and $\delta_{j}=$ $c / \omega_{j}$ is the species collisionless skin depth.

[34] As RG note, the dispersion curves shown in Figure 3 describe both "electrostatic" lower hybrid waves and "electromagnetic" whistler waves. The "electrostatic" approximation corresponds to the limit $\eta \rightarrow \infty\left(v_{\mathrm{p}}=\omega /|\mathbf{k}| \ll c\right)$ in (1) or equivalently

$$
k_{\perp} \delta_{\mathrm{e}} \gg \sqrt{2}, \quad k_{z} \delta_{\mathrm{i}} \gg 1, \quad \text { and } \quad k_{z} / k_{\perp} \ll 1
$$

in equation (3). These limits lead to the usual textbook electrostatic lower hybrid dispersion relation

$$
\omega \approx \frac{\omega_{\mathrm{i}} \Omega_{\mathrm{e}}}{\sqrt{\omega_{\mathrm{e}}^{2}+\Omega_{\mathrm{e}}^{2}}} \sqrt{1+k_{z}^{2} \mathcal{R} / k_{\perp}^{2}}
$$

where $\mathcal{R}=m_{\mathrm{i}} / m_{\mathrm{e}} \gg 1$ is the ion to electron mass ratio. The electrostatic resonance cone, determined from equation (4b) at a fixed frequency, is indicated by the thick dashed lines in Figure 3. The electrostatic dispersion relation (equation (4b)) or resonance cone is a formal asymptotic approximation to the exact CHPDR. The exact dispersion relation asymptotes to the electrostatic resonance cone within approximate range of validity for the electrostatic limit in equation (4a) and denoted by the shaded region in Figure 3. The group velocity of electrostatic lower hybrid waves on the resonance cone is

$$
\mathbf{v}_{\mathrm{g}} \equiv \frac{\partial \omega}{\partial \mathbf{k}} \approx \frac{\omega_{\mathrm{e}} \Omega_{\mathrm{e}} \sqrt{\mathcal{R}} k_{z} / k_{\perp}^{3}}{\sqrt{\left(\omega_{\mathrm{e}}^{2}+\Omega_{\mathrm{e}}^{2}\right)\left(1+k_{z}^{2} \mathcal{R} / k_{\perp}^{2}\right)}}\left(k_{\perp} \hat{z}-k_{z} \hat{\perp}\right) .
$$

[35] The phase velocity $\mathbf{v}_{\mathrm{p}}$ of electrostatic lower hybrid waves is orthogonal to the group velocity $\mathbf{v}_{\mathrm{g}}$. The perpendicular phase velocity $\mathbf{v}_{\mathrm{p} \perp}$ and perpendicular group velocity $\mathbf{v}_{\mathrm{g} \perp}$ are antiparallel $\left(\mathbf{k}_{\perp} \cdot \mathbf{v}_{\mathrm{g} \perp}<0\right)$. Consequently, lower hybrid waves are termed "backward propagating" in the plane perpendicular to the magnetic field. This "backward" relationship between the phase and group velocity is observed in both laboratory [Stenzel and Gekelman, 1975; Bellan and Porkolab, 1975; Bamber et al., 1994, 1995] and space experiments [Santolik and Gurnett, 2002].

[36] Oblique "electromagnetic" whistler waves are recovered from equation (3) by making the approximations

$$
k \delta_{\mathrm{e}} \ll \omega_{\mathrm{e}} / \Omega_{\mathrm{UH}} \text { and } k_{z} \delta_{\mathrm{i}} \gg 1
$$

which leads to the dispersion relation

$$
\omega \approx \Omega_{\mathrm{e}} k k_{z} \delta_{\mathrm{e}}^{2} .
$$

The group velocity of oblique whistler waves is

$$
v_{\mathrm{g}} \approx \frac{\Omega_{\mathrm{e}}}{k}\left[k_{\perp} k_{z} \hat{\perp}+\left(k^{2}+k_{z}^{2}\right) \hat{z}\right] \delta_{\mathrm{e}}^{2} .
$$

The perpendicular phase velocity $\mathbf{v}_{\mathrm{p} \perp}$ and perpendicular group velocity $\mathbf{v}_{\mathrm{g} \perp}$ are parallel $\left(\mathbf{k}_{\perp} \cdot \mathbf{v}_{\mathrm{g} \perp}>0\right)$. Consequently, whistler waves are termed "forward propagating" perpendicular to the magnetic field.

\subsection{Gendrin Mode}

[37] Since electrostatic lower hybrid waves are "backward" waves with $\mathbf{k}_{\perp} \cdot \mathbf{v}_{\mathrm{g} \perp}<0$ (antiparallel) and electromagnetic whistler waves are "forward" propagating waves with $\mathbf{k}_{\perp} \cdot \mathbf{v}_{\mathrm{g} \perp}>0$ (parallel), the group velocity in the perpendicular direction must pass through zero $\left(\mathbf{k}_{\perp} \cdot \partial \omega / \partial\right.$ $\mathbf{k}_{\perp}=0$ ) for some critical value denoted $\left.k_{\perp \mathrm{c}} \equiv k_{\perp}\right|_{\mathbf{v}_{\mathrm{g} \perp=0}}$, where $k_{z}\left(k_{\perp \mathrm{c}}\right)$ is a minimum for a fixed frequency. Setting the perpendicular group velocity of the full dispersion relation (3) equal to zero $\left(v_{\mathrm{g} \perp}=0\right)$ with $k_{z} \delta_{\mathrm{i}} \gg 1$ and $m_{\mathrm{e}} / m_{\mathrm{i}} \ll 1$ produces

$$
k_{\perp \mathrm{c}}^{2} \delta_{\mathrm{e}}^{2} \approx \frac{\omega_{\mathrm{e}}}{\Omega_{\mathrm{UH}}} \sqrt{1+k_{z}^{2} \delta_{\mathrm{e}}^{2}}-k_{z}^{2} \delta_{\mathrm{e}}^{2} .
$$

For $k_{z} \delta_{\mathrm{e}} \ll 1$ the critical wavenumber is on the order of the inverse electron skin depth

$$
k_{\perp \mathrm{c}} \approx \frac{1}{\delta_{\mathrm{e}}} \frac{\omega_{\mathrm{e}}}{\Omega_{\mathrm{UH}}} .
$$

This becomes $k_{\perp \mathrm{c}} \approx \delta_{\mathrm{e}}^{-1}$ in the high density limit $\omega_{\mathrm{e}} \gg \Omega_{\mathrm{e}}$. [38] RG note on p. 28,873 that the critical wavenumber can be interpreted as the dividing line between whistler $\left(k_{\perp}<k_{\perp \mathrm{c}}\right)$ and lower hybrid waves $\left(k_{\perp}>k_{\perp \mathrm{c}}\right)$. A more accurate computation of the critical wavenumber is indicated by the light dashed line in Figure 3 . This line is nearly vertical at a value given by equation (6b) over a broad spectrum of parallel wavenumbers $k_{z}$ and corresponding frequencies. Waves corresponding to $k_{\perp}=k_{\perp \mathrm{c}}$ are sometimes called the "Gendrin modes" [Gendrin, 1961]. For $m_{\mathrm{e}} / m_{\mathrm{i}} \ll 1$ and $\delta_{\mathrm{i}}^{-1} \ll k_{z} \ll \delta_{\mathrm{e}}^{-1}$ the parallel (and total) group velocity of the Gendrin modes is also constant for a broad spectrum of parallel wavenumbers $k_{z}$ and corresponding frequencies

$$
v_{\mathrm{g} \|}\left(k_{\perp \mathrm{c}}, k_{z}\right) \approx \frac{c \omega_{\mathrm{e}}}{\Omega_{\mathrm{e}}} \sqrt{2+\left(\Omega_{\mathrm{e}} / \omega_{\mathrm{e}}\right)^{2}-2 \sqrt{1+\left(\Omega_{\mathrm{e}} / \omega_{\mathrm{e}}\right)^{2}}} .
$$

This becomes $v_{g \|}\left(k_{\perp \mathrm{c}}, k_{z}\right) \approx \mathrm{c} \Omega_{\mathrm{e}} /\left(2 \omega_{\mathrm{e}}\right)$ in the high density limit $\left(\omega_{\mathrm{e}} \gg \Omega_{\mathrm{e}}\right)$ [Gendrin, 1961; Sturrock, 1962]. A wavepacket comprised of Gendrin modes will propagate in a 
homogeneous anisotropic plasma without distortion as a linear "soliton."

[39] Based on the approximations in equations (4a) and $(5 b)$ which result in the "electrostatic" lower hybrid wave and the "electromagnetic" whistler wave, the inverse electron skin depth $k_{\perp} \sim \delta_{\mathrm{e}}^{-1}$ approximately delineates the transition point between electromagnetic and electrostatic phenomena. That is not to say that waves with $k_{\perp}<\delta_{\mathrm{e}}^{-1}$ are fully electromagnetic and $k_{\perp}>\delta_{\mathrm{e}}^{-1}$ are fully electrostatic but only to say that for $k_{\perp}<\delta_{\mathrm{e}}^{-1}$ the electromagnetic fields dominate the physics and for $k_{\perp}>\delta_{\mathrm{e}}^{-1}$ the electrostatic fields dominate the physics. Discriminating between electromagnetic $k_{\perp} \delta_{\mathrm{e}} \ll 1$ and electrostatic $1 \ll k_{\perp} \delta_{\mathrm{e}}$ phenomena using the electron skin depth is not unique to lower hybrid waves. Dispersive Alfvén waves become electrostatic when $k_{\perp} \delta_{\mathrm{e}} \gg 1$ [Stasiewicz et al., 2000]. Gendrin modes, which propagate without dispersion, correspond to perpendicular wavenumbers on the order of the inverse electron skin depth $k_{\perp \mathrm{c}} \sim \delta_{\mathrm{e}}^{-1}$. For the experimental conditions investigated by $\mathrm{RG}$, the electrostatic approximation corresponds to $k_{\perp} \gg$ $1.2-1.9 \mathrm{~cm}^{-1}$ and the critical perpendicular wavenumber corresponds to about $k_{\perp \mathrm{c}} \sim 0.6-1.1 \mathrm{~cm}^{-1}$ or equivalently $k_{\perp \mathrm{c}} \delta_{\mathrm{e}} \sim 0.7-0.8$. The measured wavenumbers of the laboratory mode $k_{\perp} \sim 0.5-1.6 \mathrm{~cm}^{-1}$ are not consistent with the electrostatic limit $k_{\perp} \gg \delta_{\mathrm{e}}^{-1}$ of lower hybrid waves and are closer to the Gendrin mode of the homogeneous plasma. However, while the wavenumbers of the cavity mode in the laboratory might appear consistent with the Gendrin mode, the next section demonstrates that the field properties of the laboratory mode are profoundly inconsistent with the predictions of the CHPDR and the electrostatic approximation in equation (4b).

\section{CHPDR and Mode Identification in the Laboratory}

[40] A major theme of RG's paper is that the dispersion relation of the laboratory mode is described by "electrostatic theory," which assumes a longitudinal electric field, but that the observed electric fields are essentially transverse (electromagnetic). RG compare their laboratory measurements with the fully electromagnetic CHPDR for normal modes of a cold homogeneous plasma not the resonance cone derived from electrostatic theory. The laboratory mode is identified as an electrostatic lower hybrid wave based on the "very good agreement between the theoretical and measured values" of wavenumber [RG, p. 28,880]. Comparing the wavenumbers predicted by the CHPDR and measured in the laboratory experiment is only one way on quantifying agreement (or disagreement) between theory and observation. The theory of a cold homogeneous plasma (equation (1)) also predicts the relative size of the electric field components and corresponding longitudinal and transverse electric field vectors. Comparing the ratio of longitudinal (electrostatic) and transverse (electromagnetic) fields predicted by the CHPDR and measured in the laboratory experiment provides another means of quantifying agreement (or disagreement) between theory and observation.

\subsection{Longitudinal and Transverse Electric Fields}

[41] The term "electrostatic" seems to be a point of general confusion and contention in space physics. The electrostatic approximation "lies simply in the replacement of the vector electric field by the potential gradient $\mathbf{E}=$ $-\nabla \psi "[$ Stix, 1992, p. 54]. The electrostatic field associated with a wave is longitudinal or along the direction of $\mathbf{k}$, e.g., $\mathbf{E}_{\mathrm{L}}=-\nabla \psi$. The electromagnetic field associated with a wave is transverse or perpendicular to the direction of $\mathbf{k}$, e.g., $\mathbf{E}_{\mathrm{T}}=-\partial_{t} \mathbf{A}$ where $\mathbf{A}$ is the vector potential in the Coulomb gauge $(\nabla \cdot \mathbf{A}=0)$. Throughout this discussion the terms longitudinal and transverse will be used to describe the orientation of $\mathbf{E}$ respect to $\mathbf{k}$, and the terms perpendicular and parallel will be used to describe the orientation of $\mathbf{E}$ with respect to the background applied magnetic field $\mathbf{B}_{0}=$ $B_{0} \hat{z}$.

[42] Generally, the electric field of a plasma wave exhibits both electrostatic and electromagnetic fields. However, when either the longitudinal or transverse field dominates, the wave may be characterized as electrostatic or electromagnetic respectively in deference to the lowest order approximation that describes the essential dynamics of the mode. From this rigorous perspective, the electromagnetic or electrostatic nature of the fluctuations may be characterized solely on the basis of the electric fields without any reference to the relative or absolute size of the associated magnetic fluctuations. This characterization is important for quantifying the results of the UCLA/LAPD laboratory experiment because: (1) RG focus exclusively on the behavior of the electric field fluctuations in the laboratory experiment (2) "The electric field measurements presented are not absolutely calibrated. The anisotropic susceptibility of the plasma prevents dipole probes with a tip-to-tip separation larger than a Debye length from being calibrated [Stenzel and Fredricks, 1975]" [RG, p. 28,874]. This second point eliminates the ratio of the wave electric or magnetic field energy divided by the total wave energy as an effectual technique for characterizing the electrostatic or electromagnetic nature of the fluctuations in the laboratory experiment.

[43] The electrostatic approximation is analogous to the familiar quasineutral approximation in plasma physics. The quasineutral approximation assumes that the densities of the ions and electrons are equal everywhere to lowest order $\left(n_{\mathrm{i}}=n_{\mathrm{e}}\right)$. This does not mean that the electric field is zero. However, this assumption does mean that the Poisson equation cannot be used to determine the electric field. Once the electric field is determined by $\nabla \cdot \mathbf{J}=0$, the charge separation can be estimated from Poisson's equation. Similarly, the electrostatic approximation assumes that $\mathbf{E}=-\nabla \psi+\mathcal{O}\left(E_{\mathrm{T}} / E_{\mathrm{L}}\right)$. This approximation presumes that the electric field is predominantly longitudinal and curl free to lowest order. This does not imply that the magnetic fluctuation associated with the electrostatic wave is zero. However, this does imply that Faraday's law $\partial_{t} \mathbf{B}=-\nabla \times \mathbf{E}$ cannot be used to determine the magnetic fluctuation $\mathbf{B}$. Once the current of the electrostatic wave is determined, the associated magnetic fluctuation may be estimated from Ampere's law $\nabla \times \mathbf{B} \approx \mu_{0}\left(\mathbf{J}+\epsilon_{0} \partial_{t} \mathbf{E}_{\mathrm{L}}\right)$ where $\mathbf{J}=\mathbf{J}_{\mathrm{L}}+\mathbf{J}_{\mathrm{T}}$ is an implicit function of $\mathbf{E}_{\mathrm{L}}$ with $\nabla \cdot \mathbf{J}_{\mathrm{T}}=0$ and $\nabla \times \mathbf{J}_{\mathrm{L}}=0$. (Note that the constraint $\nabla \cdot\left(\mathbf{J}+\epsilon_{0} \partial_{t} \mathbf{E}_{\mathrm{L}}\right)=0$ does not imply that $\mathbf{J}+\epsilon_{0} \partial_{t} \mathbf{E}_{\mathrm{L}}=0$, i.e., $\mathbf{J}_{\mathrm{T}} \neq 0$ with the $\mathbf{J}_{\mathrm{L}}+\epsilon_{0} \partial_{t} \mathbf{E}_{\mathrm{L}}=0$ (longitudinal components canceling).) Subsequently, $\nabla \times \mathbf{E}$ may then be estimated from Faraday's law $\nabla \times \mathbf{E}=-\partial_{t} \mathbf{B}$. The magnetic field associated with an "electrostatic" wave 
may be generally estimated from Ampere's law [Stix, 1992, p. 78]. However, experimentally the noise levels and sensitivity of the detector determine whether the magnetic fields associated with "electrostatic" waves are observable.

[44] The longitudinal and transverse components of the electric field $\mathbf{E}=\mathbf{E}_{\mathrm{L}}+\mathbf{E}_{\mathrm{T}}$ for a wave with $\mathbf{k}=\left(k_{x}, 0, k_{z}\right)$ are

$$
\begin{array}{r}
E_{\mathrm{L}}=\frac{1}{k^{2}}\left(k_{x}^{2} E_{x}+k_{x} k_{z} E_{z}, 0, k_{z}^{2} E_{z}+k_{x} k_{z} E_{x}\right), \\
E_{\mathrm{T}}=\frac{1}{k^{2}}\left(k_{z}^{2} E_{x}-k_{x} k_{z} E_{z}, k^{2} E_{y}, k_{x}^{2} E_{z}-k_{x} k_{z} E_{x}\right) .
\end{array}
$$

[45] The electric field components are determined from equation (1a) for frequencies and wavenumbers which satisfy equation (2). The ratio $E_{\mathrm{L}} / E_{\mathrm{T}}$ is a measure of the electrostatic or electromagnetic character of a wave. For large values of this ratio, the wave is essentially electrostatic and for small values, the wave is essentially electromagnetic. Regardless of the zeroth order characterization of the fluctuations, the magnitude of the magnetic fluctuation $B$ anywhere on the whistler-lower hybrid dispersion surface of the CHPDR is simply $B=k E_{\mathrm{T}} / \omega$.

[46] Figure 4 shows the ratio of the longitudinal to transverse electric field computed from the CHPDR for conditions corresponding to the laboratory experiment. Figure $4 \mathrm{a}$ corresponds to the plasma density external to the striation $n_{0}=5 \times 10^{11} \mathrm{~cm}^{-3}$ and Figure $4 \mathrm{~b}$ corresponds to the plasma density at the minimum of the striation $n_{0}=$ $2 \times 10^{11} \mathrm{~cm}^{-3}$. Since a magnetized plasma is anisotropic, the ratio is computed for the electric field perpendicular to and parallel to the applied magnetic field $\mathbf{B}_{0}=B_{0} \hat{z}$. The upper and lower solid lines correspond to the perpendicular ratio $\left|E_{\mathrm{L} \perp} / E_{\mathrm{T} \perp}\right|$ for the two frequencies $f=30 \mathrm{MHz}$ and $f=90 \mathrm{MHz}$, respectively. The dashed lines correspond to the parallel ratio $\left|E_{\mathrm{L} z} / E_{\mathrm{T} z}\right|$ which is essentially the same for both frequencies on this scale.

[47] There is a clear difference between the behavior of the parallel $\left|E_{\mathrm{L} z} / E_{\mathrm{T} z}\right|$ and perpendicular $\left|E_{\mathrm{L} \perp} / E_{\mathrm{T} \perp}\right|$ ratios. The parallel ratio behaves as

$$
\left|E_{\mathrm{L} z} / E_{\mathrm{T} z}\right| \approx 1+k^{2} \delta_{\mathrm{e}}^{2} .
$$

This ratio becomes electrostatic for $k_{\perp} \sim k \gtrsim \delta_{\mathrm{e}}^{-1}$ with $k_{z} / k_{\perp} \ll 1$, and the inflection point in Figure 4 occurs at $k_{\perp}$ $\sim k=\delta_{\mathrm{e}}{ }^{-1}$. In contrast, the perpendicular ratio $\left|E_{\mathrm{L} \perp}\right| E_{\mathrm{T} \perp} \mid$ remains large down to very small wavenumbers, and the electric field perpendicular to the magnetic field is essentially longitudinal (electrostatic) despite the important electromagnetic corrections to the parallel dynamics and the overall dispersion relation. The whistler-lower-hybrid dispersion relation is "electromagnetic" for $k_{\perp} \lesssim \delta_{\mathrm{e}}^{-1}$ because the electron current along the magnetic field is significant; the parallel component of the vector potential $A_{z}$ is no longer negligible in comparison with the scalar potential $\partial_{t} A_{z} \sim \partial_{z} \psi$. Thus for wavenumbers $k_{\perp} \lesssim \delta_{\mathrm{e}}^{-1}$ the parallel vector potential is essential to the wave dynamics and the dispersion relation. However, the perpendicular vector potential $\mathbf{A}_{\perp}$ is negligible unless $k_{\perp} \ll \delta_{\mathrm{e}}^{-1}$.

[48] Sections 4.1, 4.2, and 5.1 demonstrate that the electron skin depth $\delta_{\mathrm{e}}$ is the characteristic scale length at
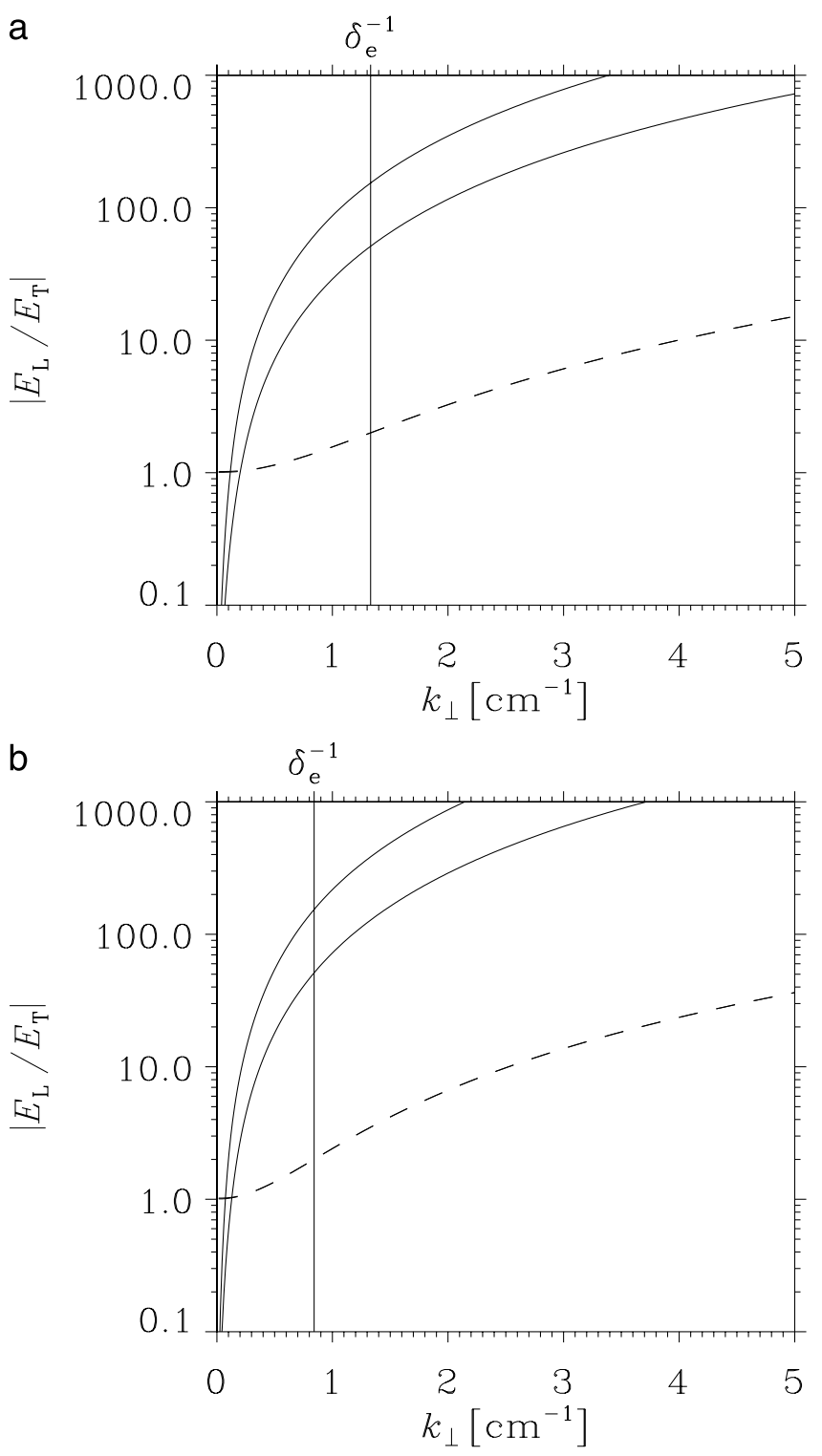

Figure 4. Ratio of the longitudinal to transverse electric field computed from the CHPDR for conditions corresponding to the laboratory experiment with $n_{0}=5 \times 10^{11} \mathrm{~cm}^{-3}$ (a) and $n_{0}=2 \times 10^{11} \mathrm{~cm}^{-3}$ (b) for lower hybrid waves with $f=30 \mathrm{MHz}$ (upper solid lines) and $f=90 \mathrm{MHz}$ (lower solid lines). The solid lines correspond to the ratio $\left|E_{\mathrm{L} \perp} / E_{\mathrm{T} \perp}\right|$ and the dashed lines correspond to the ratio $\left|E_{\mathrm{L} z} / E_{\mathrm{T} z}\right|$. The latter ratio appears the same for both frequencies on this scale.

which electromagnetic whistlers transition to electrostatic lower hybrid waves using three different but related criteria: (1) The "electromagnetic" whistler dispersion relation is valid for $k_{\perp} \ll \delta_{\mathrm{e}}^{-1}$ and the "electrostatic" lower hybrid dispersion relation is valid for $k_{\perp} \gg \delta_{\mathrm{e}}^{-1}$. (2) These two modes are separated by the Gendrin mode which occurs at $k_{\perp}=k_{\perp \mathrm{c}} \sim \delta_{\mathrm{e}}^{-1}$. (3) The ratios $\left|E_{\mathrm{L} z} / E_{\mathrm{T} z}\right|$ and $\left|E_{\mathrm{L} \perp} / E_{\mathrm{T} \perp}\right|$ are both large for $k_{\perp}>\delta_{\mathrm{e}}^{-1}$. However, the ratio $\left|E_{\mathrm{L} z} / E_{\mathrm{T} z}\right|$ is order one for $k_{\perp}<\delta_{\mathrm{e}}^{-1}$. Since the CHPDR does not model the propagation of waves in a density gradient, the above calculations must be considered estimates for the laboratory plasma which is extremely inhomogeneous with sharp 
Table 4. Perpendicular Wavenumber Estimates From the Three Density Striations Investigated by $\mathrm{RG}^{\mathrm{a}}$

\begin{tabular}{ccc}
\hline Striation & $k_{\perp}, \mathrm{cm}^{-1}$ & From RG \\
\hline 1 & $0.5-1.6$ & Figure 7 \\
2 & 1.2 & Figure 5 \\
3 & 4 & Figure 6 \\
\hline
\end{tabular}

${ }^{\mathrm{a} O n l y}$ striation 3 exhibits wavenumbers which are in the electrostatic regime $k_{\perp}>\sqrt{2} \delta_{\mathrm{e}}^{-1} \sim 2 \mathrm{~cm}^{-1}$ for the homogeneous plasma.

density gradients. Nonetheless, for the whistler-lowerhybrid dispersion relation we generally expect electrostatic behavior for wavenumbers $k_{\perp} \gg \delta_{\mathrm{e}}^{-1}$.

\subsection{Comparison With the Laboratory Experiment}

[49] The theoretical results presented above generally disagree with RG's interpretation of the laboratory data. On p. 28,882, RG state that 'though lower hybrid waves' dispersion can be well described by electrostatic theory, wave magnetic fields play an important role in the physics of these waves." However, we have demonstrated that electrostatic theory implies, by definition, that the transverse electric field and associated magnetic field contribute minimally to the dynamics of electrostatic waves; these electromagnetic fields are mere corrections to the electrostatic dispersion relation in the appropriate limit in equation (4a). RG also claim that the "measured $k$-spectra for the laboratory experiments show that the laboratory data satisfy any reasonable requirements about what constitutes an electrostatic wave." However, the laboratory data does not satisfy the usual criteria used to establish that a wave is electrostatic. Plate 2, on p. 28,869 of RG, shows that the perpendicular electric field in the laboratory experiment is essentially transverse (nonzero curl) with $E_{\mathrm{T} \perp} \gg E_{\mathrm{L} \perp}$ whereas an electrostatic wave exhibits an electric field which is essentially longitudinal with $E_{\mathrm{L} \perp} \gg E_{\mathrm{T} \perp}$. Furthermore, the measured $k$-spectra in the laboratory are generally not consistent with the electrostatic regime nor do they agree with the electrostatic dispersion relation denoted by the dashed lines in Figure 3. The electrostatic dispersion relation differs significantly from the CHPDR near $k_{\perp} \sim 1 \mathrm{~cm}^{-1}$, both in the wavenumbers and in the magnitude and direction of the group velocity. Fluctuations with $k_{\perp} \sim 1 \mathrm{~cm}^{-1}$ can hardly be deemed consistent with the electrostatic dispersion relation (the resonance cone). The wavenumbers exhibited by the three density striations investigated by $\mathrm{RG}$ are summarized in Table 4. Only striation 3 exhibits wavenumbers which are even marginally in the electrostatic regime $k_{\perp}=4 \mathrm{~cm}^{-1} \gtrsim \sqrt{2} \delta_{\mathrm{e}}^{-1} \sim$ $2 \mathrm{~cm}^{-1}$ for the homogeneous plasma. We emphasize that there may be some differences in the boundaries of the electrostatic regime due to the extreme plasma inhomogeneities investigated in the laboratory experiment. Nevertheless, we expect that the the fluctuations to exhibit electrostatic properties when $k_{\perp} \delta_{\mathrm{e}} \gg 1$.

[50] Figure 4 demonstrates that the CHPDR predicts that the perpendicular electric field for the whistler-lower-hybrid mode is essentially "electrostatic" with $\left|E_{\mathrm{L} \perp} / E_{\mathrm{T} \perp}\right| \sim 20-$ 2000 for wavenumbers $k_{\perp}=1-4 \mathrm{~cm}^{-1}$ and plasma conditions corresponding to the laboratory experiment. However, the perpendicular electric field in the laboratory is both locally $(\mathbf{E} \perp \mathbf{k})$ and globally $(\oint \mathbf{E} \cdot d \mathbf{l} \neq 0)$ transverse or electromagnetic with a ratio $\left|E_{\mathrm{L} \perp} / E_{\mathrm{T} \perp}\right| \sim 10^{-1}$. Indeed, $\mathrm{RG}$ state in the discussion (on p. 28,879) of Plate 2 that for the laboratory mode the "the field pattern is not electrostatic, i.e., $\nabla \times \mathbf{E} \neq 0, \mathbf{A} \neq 0$; a closed path integral of the electric field is not zero." The theoretical predictions of the CHPDR and the laboratory measurements conservatively differ by $2-4$ orders of magnitude on this point.

[51] The laboratory results are not well described by the CHPDR nor are the wavenumbers consistent with the electrostatic dispersion relation (the resonance cone). The evidence strongly suggests that the laboratory mode cannot be described by homogeneous plasma theory. This is not surprising since the mode appears to be localized in a plasma density gradient, and the physics of a plasma density gradient is not described by homogeneous plasma theory. This is one point where the space observations and laboratory measurements appear to be in agreement. The theories most consistent with LHSS phase velocity estimates are based on inhomogeneous plasma theory; the Hall current in a density gradient produced by the $\mathbf{E} \times \mathbf{B}_{0}$ response of the electrons and the inertial response of the ions [Seyler, 1994; Schuck et al., 1998; Schuck, 1999; Schuck and Bonnell, 2003]. Indeed, Schuck and Bonnell [2003] point out that ray-tracing descriptions of LHSS based on the CHPDR consistently fail to describe the frequencies and phase velocity estimates of LHSS waves [Pinçon et al., 1997; Schuck et al., 1998; Bonnell et al., 1998; Pécseli et al., 1996, 1997; Kjus et al., 1998; Høymork et al., 2000, 2001]. The theories of LHSS do not describe the quasi-electromagnetic regime $k_{\perp} \delta_{\mathrm{e}} \lesssim 1$ of the laboratory measurements [Seyler, 1994; Schuck et al., 1998; Schuck, 1999; Schuck and Bonnell, 2003]. However, these theories were derived for the electrostatic regime $k_{\perp} \delta_{\mathrm{e}} \gg 1$ corresponding to the scale lengths observed in space, and in the appropriate regime these theories appear to provide an adequate description of LHSS wave fields [Pinçon et al., 1997; Schuck et al., 1998; Bonnell et al., 1998; Tjulin et al., 2003].

[52] There is another significant but less profound discrepancy between the CHPDR and the laboratory measurements. In Figure 5 on p. 28,879, RG compare the CHPDR and the measured $k_{z}$ and $k_{\perp}$ distributions in striation 2 and claim "very good agreement between the theoretical and measured values" [RG, p. 28,880]. In our opinion, the agreement does not appear to be very good. Given the measured $k_{z}$-spectrum, the CHPDR (which is double valued at $k_{z}=0.027 \mathrm{~cm}^{-1}$ ) would predict a bimodal $k_{\perp}$-spectrum, with both small and large wavenumbers represented at roughly 0.2 and $2.2 \mathrm{~cm}^{-1}$, respectively. Instead the observed $k_{\perp}$-spectrum is basically unimodal with peak power at $k_{\perp}=1.2 \mathrm{~cm}^{-1}$; this is inconsistent with the measured $k_{z}$-spectrum and the CHPDR. A referee of this paper notes that a small error in the $k_{z}$-spectrum would lead to a $k_{\perp}$-spectrum with a single maximum centered at the minimum of the $k_{z}\left(k_{\perp}\right)$ dispersion relation. While this supposition might bring the laboratory measurements into agreement with the CHPDR, the resulting $k$-spectrum now appears to correspond to a Gendrin mode and not an electrostatic lower hybrid wave with wavenumbers near the resonance cone denoted by the dashed lines in Figure 3 . We point out that that regardless of the apparent agreement or disagreement between the CHPDR and the $k$-spectrum measured in the laboratory, the predicted and measured electric field 
patterns disagree profoundly. (The comparison between the CHPDR and the measured $k$-spectrum is further complicated by the variation in the density profile along $y=0 \mathrm{~cm}$ from shot to shot. Plates 1 and 4 of $\mathrm{RG}$ indicate that the background plasma density varies by $20 \%$ between measurements for the same striation. The estimate of $20 \%$ presumes that the label on the right-hand side of the bottom panel in Figure 4 should read $\left(\times 10^{11} \mathrm{~cm}^{-3}\right)$ and not $\left(\times 10^{12} \mathrm{~cm}^{-3}\right)$.)

\section{Summary and Conclusion}

[53] RG observe enhanced electric fields localized in gradients of plasma density depletions at frequencies near the lower hybrid resonance. The electric field points along lines of constant density and is both locally and globally electromagnetic (transverse) regardless of the striation shape. The perpendicular wavenumber of the laboratory modes is set by the diameter of the density striation, and this scaling is independent of frequency and striation size. RG's observations of electromagnetic fluctuations localized in a density gradient are new, extremely interesting, and possible relevant to some other ionospheric observations. However, the apparent correspondence between the laboratory data and ionospheric phase velocity estimates of LHSS [Pinçon et al., 1997; Schuck et al., 1998; Bonnell et al., 1998] is irrelevant as the laboratory and ionospheric experiments perform inherently different measurements involving different combinations of electric field components and interferometric phase velocity estimations relative to the trajectory. Furthermore, the phase velocity derived from an idealized laboratory phase distribution is inconsistent with the ionospheric measurements; the results are opposite to that observed in space. The evidence presented by RG suggests that the laboratory and ionospheric data correspond to entirely different phenomena. Additionally, we disagree with RG's claim that the laboratory measurement agree with the theoretical predictions of the cold homogeneous plasma dispersion relation (CHPDR). The perpendicular electric field in RG's observations is mainly transverse whereas the CHPDR predicts that it should be essentially longitudinal for the frequencies and wavenumbers measured in the laboratory. In conclusion, RG's laboratory measurements are not relevant to ionospheric observations of LHSS in the work of Pinçon et al. [1997], Schuck et al. [1998], and Bonnell et al. [1998]. Nor are the data relevant to more recent magnetospheric measurements of LHSS which confirm of these ionospheric observations [Tjulin et al., 2003]. However, we generally agree that laboratory experiments provide an important complement to spacecraft observations and feel that future collaborative work between the space and laboratory community would be profitable.

[54] Acknowledgments. The authors acknowledge useful discussions concerning the laboratory measurements with William E. Amatucci, the Alaska '93 data set with Gregory T. Delory, and the Altair-AppletonHartree dispersion relation with Robert Strangeway. Additionally, the authors would like to thank the referees for providing useful criticisms and for suggesting that this "comment" should become a full-length journal article.

[55] Shadia Rifai Habbal thanks both referees for their assistance in evaluating this paper.

\section{References}

Appleton, E. V. (1928), The influence of the earth's magnetic field on wireless transmission (summary), in U.R.S.I. Proc. 1927, Brussels, Washington Assembly.

Appleton, E. V. (1932), Wireless studies of the ionosphere, J. Inst. Elec. Eng., 71, 642 .

Arnoldy, R., K. Lynch, P. M. Kintner, J. Vago, S. Chesney, T. E. Moore, and C. J. Pollock (1992), Bursts of transverse ion acceleration at rocket altitudes, Geophys. Res. Lett., 19(4), 413-416.

Bamber, J. F., W. Gekelman, and J. E. Maggs (1994), Whistler wave mode conversion to lower hybrid waves at a density striation: A laboratory investigation of an auroral process, Phys. Rev. Lett, 73(22), 2990-2993.

Bamber, J. F., J. E. Maggs, and W. Gekelman (1995), Whistler wave interaction with a density striation: A laboratory investigation of an auroral process, J. Geophys. Res., 100(12), 23,795-23,810.

Bellan, P., and M. Porkolab (1975), Excitation of lower hybrid waves by a slow wave structure, Phys. Rev. Lett., 34(3), 124-127.

Bonnell, J. W., P. W. Schuck, J.-L. Pinçon, C. E. Seyler, and P. M. Kintner (1998), Observation of bound states and counter-rotating eigenmodes in the auroral ionosphere, Phys. Rev. Lett., 80(26), 5734-5737.

Delory, G. T. (1996), Rocket observations of vlf waves, electron precipitation and ion heating in the auroral ionosphere, Ph.D. thesis, University of California, Berkeley, Berkeley, Calif.

Delory, G., P. Schuck, C. Seyler, R. Ergun, C. Carlson, and J. McFadden (1998), Measurements of auroral VLF bursts as rotating eigenmode structures on the alaska '93 sounding rocket, Eos Trans. $A G U, 79(17)$, Spring Meet. Suppl., S110.

Dovner, P. O., A. I. Eriksson, R. Boström, and B. Holback (1994), Freja multiprobe observations of electrostatic solitary structures, Geophys. Res. Lett., 21(27), 1827-1834.

Dupree, T. H. (1982), Theory of phase-space density holes, Phys. Fluids, 25(2), 277-289.

Ergun, R. E., E. M. Klementis, G. T. Delory, C. W. Carlson, and J. P. McFadden (1994), Plasma density observations during large-amplitude lower hybrid emissions, Eos Trans. $A G U, 75(44)$, Fall Meet. Suppl., F552-F553.

Ergun, R. E., E. M. Klemetis, G. T. Delory, J. P. McFadden, and C. W. Carlson (1995), VLF wave localization in the low-altitude auroral region, Geophy. Res. Lett., 22(16), 2099-2102.

Ergun, R. E., et al. (1998a), FAST satellite observations of large-amplitude solitary structures, Geophys. Res. Lett., 25(12), 2041-2044.

Ergun, R. E., C. W. Carlson, J. P. McFadden, F. S. Mozer, L. Muschietti, and I. Roth (1998b), Debeye-scale plasma structures associated with magnetic field aligned electric fields, Phys. Rev. Lett., 81(4), 826-829.

Eriksson, A. I., B. Holback, P. O. Dovner, R. Boström, G. Holmgren, M. André, L. Eliasson, and P. M. Kintner (1994), Freja observations of correlated small-scale density depletions and enhanced lower hybrid waves, Geophys. Res. Lett., 21(27), 1843-1846.

Franz, J. R., P. M. Kintner, and J. Pickett (1998), POLAR observations of coherent electric field structures, Geophys. Res. Lett, 25(8), 1277-1280.

Franz, J. R., P. M. Kintner, C. E. Seyler, J. Pickett, and J. D. Scudder (2000), On the perpendicular scale of electron phase-space holes, Geophys. Res. Lett, 27(2), 172-179.

Gendrin, R. (1961), Le guidage des whistlers par le champ magnetique, Planet. Space Sci., 5(4), 274-282.

Gillmor, C. S. (1982), Wilhelm Altar, Edward Appleton, and the magnetoionic theory, Proc. Am. Philos. Soc., 126, 395-440.

Hartree, D. R. (1931), The propagation of electromagnetic waves in a refracting medium in a magnatic field, Proc. Cambridge Philos. Soc., 27, 97-120.

Høymork, S. H., H. L. Pécseli, B. Lybekk, J. Trulsen, and A. Eriksson (2000), Cavitation of lower hybrid waves in the earth's ionosphere: A model analysis, J. Geophys. Res., 105(A8), 18,519-18,535.

Høymork, S. H., H. L. Pecseli, B. Lybekk, J. Trulsen, and A. Eriksson (2001), The shape and evolution of lower hybrid density cavities observed by FREJA, Phys. Chem. Earth, Part C, 26(1-3), 213-217.

Inuzuka, H., Y. Takeda, and K. Yamagiwa (1997), Observation of lower hybrid wave packets with very large amplitude in a high-voltage linear plasma discharge, Phys. Lett. A, 234(3), 219-224.

Kintner, P. M., J. Vago, S. Chesney, R. L. Arnoldy, K. A. Lynch, C. J. Pollock, and T. E. Moore (1992), Localized lower hybrid acceleration of ionospheric plasma, Phys. Rev. Lett., 68(16), 2448-2451.

Kjus, S. H., H. L. Pécseli, B. Lybekk, J. Holtet, J. Trulsen, H. Lür, and A. Eriksson (1998), Statistics of the lower hybrid wave cavities detected by the freja satellite, J. Geophys. Res., 103(A11), 26,633-26,647.

Knudsen, D. J., J. H. Clemmons, and J.-E. Wahlund (1998), Correlation between core ion energization, suprathermal electron bursts, and broadband ELF plasma waves, J. Geophys. Res., 103(A3), 4171-4186.

Knudsen, D. J., D. D. Wallis, and H. G. James (1999), Tethered two-point measurements of solitary auroral density cavities, Geophys. Res. Lett., 26(19), 2933-2936. 
Kostrov, A. V., A. V. Strikovskiy, and A. V. Shashurin (2001), Plasma turbulence near the lower hybrid resonance (in Russian), Fiz. Plasmy, 27(2), 149-154 (Plasma Phys. Rep., Engl. Transl., 27(2), 137-142, 2001).

Krall, N. A., and A. W. Trivelpiece (1973), Principles of Plasma Physics, Int. Ser. in Pure and Appl. Phys., McGraw-Hill, New York.

Labelle, J., P. M. Kintner, A. W. Yau, and B. A. Whalen (1986), Largeamplitude wave packets observed in the ionosphere in association with transverse ion acceleration, J. Geophys. Res., 91(A6), 7113-7118.

Lynch, K. A., R. L. Arnoldy, P. M. Kintner, and S. Chesney (1996), Lower hybrid spikelets: Auroral butterflies or magnetospheric thermometers, in Proceeding of the 1995 Cambridge Workshop on Space Plasma Interactions, Bermuda, edited by T. Chang, p. 339, MIT Cent. for Space Res. Cambridge, Mass.

Matsumoto, H., H. Kojima, T. Miyatake, Y. Omura, M. Okada, I. Nagano, and M. Tsutsui (1994), Electrostatic solitary waves (ESW) in the magnetotail-BEN wave-forms observed by Geotail, Geophys. Res. Lett., 21(25), 2915-2918

McAdams, K. L., J. Labelle, P. W. Schuck, and P. M. Kintner (1998), PHAZE II observations of lower hybrid burst structures occurring on density gradients, Geophys. Res. Lett., 25(16), 3091-3094.

Pécseli, H. L., K. Iranpour, O. Holter, B. Lybekk, J. Holtet, J. Trulsen, A. Eriksson, and B. Holback (1996), Lower-hybrid wave cavities detected by the Freja-satellite, J. Geophys. Res., 101(8), 5299.

Pécseli, H. L., B. Lybekk, J. Trulsen, and A. Eriksson (1997), Lower-hybrid wave cavities detected by instrumented spacecraft, Plasma Phys. Controlled Fusion, 39(5A), 236-277.

Pinçon, J.-L., P. M. Kintner, P. W. Schuck, and C. E. Seyler (1997) Observation and analysis of lower hybrid solitary structures as rotating eigenmodes, J. Geophys. Res., 102(8), 17,283-17,296.

Poeverlein, H. (1948), Ray paths of radio waves in the ionosphere, Sitzungsber. Bayer. Akad. Wiss. Math. Naturwiss. Kl., 78, 175.

Rosenberg, S., and W. Gekelman (1998), Electric field measurements of directly converted lower hybrid waves at a density striation, Geophys. Res. Lett., 25(6), 865-868.

Rosenberg, S., and W. Gekelman (2001), A three-dimensional study of lower hybrid wave interactions with field-aligned density depletions, J. Geophys. Res., 106(A12), 28,867-28,884.

Santolík, O., and D. A. Gurnett (2002), Propagation of auroral hiss at high altitudes, Geophys. Res. Lett., 29(10), 1481, doi:10.1029/ 2001 GL013666.

Schuck, P. W. (1999), A theory of lower hybrid solitary structures: Rotating helical eigenstates excited by wave scattering, Ph.D. thesis, Cornell Univ., Ithaca, N.Y.
Schuck, P. W., and J. W. Bonnell (2003), Ray trajectories of lower hybrid solitary structures, J. Geophys. Res., 108(A5), 1175, doi:10.1029/ 2002JA009716.

Schuck, P. W., C. E. Seyler, J.-L. Pinçon, J. W. Bonnell, and P. M. Kintner (1998), Theory simulation and observation of bound states associated with lower hybrid solitary structures, J. Geophys. Res., 103(A4), 6935-6953.

Schuck, P. W., G. I. Ganguli, and P. M. Kintner (2002), On the role of lower hybrid collapse in the auroral ionosphere, Phys. Rev. Lett., 89(6)

Seyler, C. E. (1994), Lower hybrid wave phenomena associated with density depletions, J. Geophys. Res., 99(A10), 19,513-19,525.

Stasiewicz, K., et al. (2000), Small scale Alfvénic structure in the aurora, Space Sci. Reviews, 92(1-2), 423-533

Stenzel, R. L., and R. W. Fredricks (1975), Study of the performance of antennas in magnetized plasmas, final report, Tech. Rep., Marshall Space Flight Cent., Huntsville, Ala.

Stenzel, R. L., and W. Gekelman (1975), Electrostatic waves near the lower hybrid resonance, Phys. Rev. A, 11(6), 2057-2060.

Stix, T. H. (1992), Waves in Plasmas, Am. Inst. of Physics, New York.

Sturrock, P. A. (1962), Generation of radio noise in the vicinity of the earth, J. Res. Natl. Bur. Stand. U.S. Sect. D, 66, 153-157.

Takeda, Y., and H. Inuzuka (2000), Anomalous resistivity caused by nonlinear lower hybrid waves in a high-voltage linear plasma discharge, Phys. Lett. A, 265(4), 282-287.

Tjulin, A., A. I. Eriksson, and M. André (2003), Lower hybrid cavities in the inner magnetosphere, Geophys. Res. Lett., 30(7), 1364, doi:10.1029/ 2003GL016915.

Vago, J. L., P. M. Kintner, S. W. Chesney, R. L. Arnoldy, K. A. Lynch, T. E. Moore, and C. J. Pollock (1992), Transverse ion acceleration by localized lower hybrid waves in the topside auroral ionosphere, J. Geophys. Res., 97(A11), 16,935-16,957.

J. W. Bonnell, Space Sciences Laboratory, Centennial Dr. at Grizzly Peak Blvd., University of California, Berkeley, Berkeley, CA 94720-7450, USA. (jbonnell@ssl.berkeley.edu)

J.-L. Pinçon, LPCE/CNRS, 3A Avenue de la Recherche Scientifique, 45071 Orleans Cedex 2, France. (jlpincon@cnrs-orleans.fr)

P. W. Schuck, Plasma Physics Division, Naval Research Laboratory, Code 6794, 4555 Overlook Ave., SW, Washington, DC 20375-5346, USA. (schuck@ppdmail.nrl.navy.mil) 